\title{
The Nonlinear Geometry of Banach Spaces
}

\author{
Nigel J. KALTON
}

\author{
Department of Mathematics \\ University of Missouri-Columbia \\ Columbia, MO 65211 \\ nigel@math.missouri.edu
}

Received: November 5, 2007

Accepted: January 14, 2008

\begin{abstract}
We survey some of the recent developments in the nonlinear theory of Banach spaces, with emphasis on problems of Lipschitz and uniform homeomorphism and uniform and coarse embeddings of metric spaces.

Key words: Banach space, nonlinear, Lipschitz, uniform homeomorphism, coarse embedding.

2000 Mathematics Subject Classification: 46B25, 46 T99.
\end{abstract}

The author was supported by NSF grant DMS-0555670. 


\section{Contents}

$\begin{array}{ll}\text { Introduction } & 9\end{array}$

1. Preliminaries $\quad 11$

1.1. Basic Banach space theory . . . . . . . . . . . . . . . . 11

1.2. Homeomorphisms and isometries between Banach spaces . . . . . . . 13

1.3. Various categories of homeomorphisms . . . . . . . . . . . . . . 14

2. Lipschitz and uniform homeomorphisms between Banach $\begin{array}{ll}\text { spaces } & \mathbf{1 8}\end{array}$

2.1. Classical differentiability results for Lipschitz maps . . . . . . . . . . 18

2.2. The Lipschitz isomorphism problem, I . . . . . . . . . . . . . . . . . . 22

2.3. The Lipschitz isomorphism problem, II . . . . . . . . . . . . . . . . 26

2.4. Uniformly and coarsely homeomorphic Banach spaces . . . . . . . . . 30

3. Properties of metric spaces and extension of Lipschitz maps $\quad 35$

3.1. Nonlinear type and cotype . . . . . . . . . . . . . . . . 35

3.2. The structure of the Arens-Eells space of a metric space . . . . . . . . 39

3.3. Extension of Lipschitz maps: absolute Lipschitz retracts . . . . . . . . 41

3.4. Extending Lipschitz maps into Banach spaces . . . . . . . . . . . 42

4. Uniform and coarse embeddings 48

4.1. Uniform and coarse embeddings in $L_{p}$-spaces . . . . . . . . . . . . . . 48

4.2. Uniform and coarse embeddings of metric spaces . . . . . . . . . . 51 


\section{Introduction}

A Banach space is, by its nature, also a metric space. When we identify a Banach space with its underlying metric space, we choose to forget its linear structure. The fundamental question of nonlinear geometry is to determine to what extent the metric structure of a Banach space already determines its linear structure. This question, as stated, is somewhat ambiguous: we need to specify what information we are given about the metric space. Thus there are several variants of this problem and we will discuss them in turn. Of course this problem is not the only interest of the nonlinear theory. Many similar questions have been considered concerning nonlinear embeddings of one Banach space into another, and more generally of metric spaces into Banach spaces.

The subject is an old one, dating back almost to the origins of Banach space theory. Perhaps the first result in the area is the celebrated Mazur-Ulam theorem on isometries from 1932 [100]. Later the work of Lindenstrauss [94] and Enflo [46-48] in the 1960's gave a tremendous impetus to the study of Banach spaces as metric spaces. The explosion of interest in the linear theory of Banach spaces between 1960 and 2000, spurred some significant advances in nonlinear theory, but still the nonlinear theory seems to have played a junior role. However since about 2000, there seems to have been quite marked increase in activity in this area. There are several reasons for this. First there is the appearance of the authoritative book of Benyamini and Lindenstrauss, [16]. This book finally gave a definitive form to the subject and highlighted both what we know and what we do not know. Then there has been an upsurge of interest from other areas of mathematics in the problem of determining how well a metric space $(M, d)$ can be embedded in a particular type of Banach space. This sort of problem is of interest to theoretical computer scientists (see, e.g., [26]) in connection with data-mining and to specialists in $C^{*}$-algebras in connection with the Novikov conjecture (see, e.g., [87]). Problems of this type are very much in the spirit of nonlinear Banach space theory as we have already observed.

Thus this seems to be the beginning of a golden era for nonlinear Banach space theory. My aim in this survey is to both present some aspects of the theory which are of special interest to me and to emphasize new developments since the appearance of $[16]$

We have divided this survey into four sections. The first section is concerned with 
the preliminaries. We describe the background Banach space theory we will need in $\S 1.1$. In $\S 1.2$ we discuss some earlier work on homeomorphisms and isometries, which may be regarded as the two extremes for the type of problem we are interested in. In $\S 1.3$ we describe the general nature of embeddings and isomorphisms between metric spaces which are of interest to us.

In the second section ( $\S 2.1$ to $\S 2.4)$ we discuss the main problems: when are two Lipschitz-isomorphic or uniformly homeomorphic Banach spaces linearly isomorphic? In $\S 2.1$ we discuss the classical approach to the Lipschitz isomorphism problem (and Lipschitz embedding problems) for Banach spaces via differentiability theorems. These results are now almost classical and very well covered in [16]; nevertheless these ideas are so central that we feel that it is important for the reader to appreciate what can be done through this approach. In $\S 2.2$ and $\S 2.3$ we discuss the Lipschitz isomorphism problem; we place some degree of emphasis on the approach through Arens-Eells spaces [53] which we feel gives some new insights. In $\S 2.4$ we discuss the uniform homeomorphism problem for Banach spaces; here the methods employed lean heavily on the theory of Lipschitz maps.

In the third section, ( $\S 3.1$ to $§ 3.4)$ the theme is to consider Lipschitz properties of metric spaces which provide nonlinear analogues of well-known (linear) properties of Banach spaces. It seems to the author that it is, in fact, quite remarkable that the linear theory can be extended so successfully. There have been many spectacular advances in this area, particularly associated with the work of Naor and his collaborators. In $§ 3.1$ the problem is to extend the definitions of Rademacher type and cotype to an arbitrary metric space. In the case this was done by Enflo 35 years ago, and several other variants have been proposed; however the right definition for cotype is a very recent advance by Mendel and Naor [104]. We then discuss the structure of the Arens-Eells space associated to a metric space and consider the how this refelcts the properties of the metric space. In $\S 3.3$ we discuss some recent results on absolute Lipschitz retracts and related problems. In $\S 3.4$ we consider a remarkable circle ideas about the generalization of the Maurey extension property to metric spaces; this is another area where some great advances have recently been recorded.

In the fourth section, ( $\$ 4.1$ to $\S 4.2)$, we consider some very different problems concerning coarse and uniform embeddings of metric spaces and uniform homeomorphisms between the unit balls of Banach spaces. Here the techniques are very different and Lipschitz maps no longer play such a prominent role. In $\S 4.1$ we discuss uniform and coarse embeddings into $L_{p}$-spaces, and the problem of when the unit ball of a Banach space is uniformly homeomorphic to the unit ball of a Hilbert space. In $\S 4.2$ we discuss some other recent results on embeddings of metric spaces into certain Banach spaces.

Throughout the paper, we will state open Problems which we feel are especially important and whose solution would represent a significant advance in the field. 


\section{Preliminaries}

In this section, we will gather together some of the necessary preliminary information on Banach spaces and metric spaces which we will use later in the paper.

\subsection{Basic Banach space theory}

In this section we collect together some of the definitions which we will employ later in the paper. For reasons which we discuss in $\S 1.2$ all Banach spaces will in general be real unless otherwise stated. (There will be occasional exceptions.) If $X$ is a Banach space we write $B_{X}$ for the closed unit ball $B_{X}=\{x:\|x\| \leq 1\}$ and $\partial B_{X}$ for the unit sphere $\{x:\|x\|=1\}$. We also use $[A]$ for the closed linear span of a subset $A$ of a Banach space. The Banach-Mazur distance between two isomorphic Banach spaces $X$ and $Y$ is defined by

$$
d(X, Y)=\inf \left\{\|T\|\left\|T^{-1}\right\|: T: X \rightarrow Y \text { is an isomorphism }\right\} .
$$

We recall that a separable Banach space $X$ has the Bounded Approximation Property (BAP) if there is a sequence of finite-rank operators $T_{n}: X \rightarrow X$ so that $x=\lim _{n \rightarrow \infty} T_{n} x$ for each $x \in X$. The space $X$ is said to have the Metric Approximation Property (MAP) if we can take $\left\|T_{n}\right\|=1$ for all $n$. We refer to [31] for a full discussion of approximation properties.

We next recall the definition of the Radon-Nikodym Property. One definition of the Radon-Nikodym Property (RNP) is as follows: $Y$ has the (RNP) if and only if every Lipschitz map $f:[0,1] \rightarrow Y$ is differentiable a.e. The Radon-Nikodym Property was the center of much research in the 1970's (see [41] for example) and is now almost completely understood. In particular a reflexive Banach space has (RNP) and every separable dual space has (RNP). The most elementary non-RNP spaces are $c_{0}$ and $L_{1}$.

A Banach space $X$ is said to be finitely representable in a Banach space $Y$ if there is a constant $\lambda \geq 1$ such that for every finite-dimensional subspace $E$ of $X$ there is a linear map $T: E \rightarrow Y$ with

$$
\|x\| \leq\|T x\| \leq \lambda\|x\|, \quad x \in E .
$$

Equivalently, $X$ is finitely representable in $Y$ if, for some $\lambda \geq 1$, and for every finitedimensional subspace $E$ of $X$ there is a finite-dimensional subspace $F$ of $Y$ with $d(E, F) \leq \lambda$. Let us recall that if $X$ is a Banach space and $\mathcal{U}$ is a non-principal ultrafilter on $\mathbb{N}$ then the ultraproduct $X_{\mathcal{U}}$ is the quotient of $\ell_{\infty}(X)$ by the subspace $c_{0, \mathcal{U}}(X)$ of all sequences $\left(x_{n}\right)_{n=1}^{\infty}$ such that $\lim _{n \in \mathcal{U}}\left\|x_{n}\right\|=0$. This can be thought as the space $\ell_{\infty}(X)$ equipped with the semi-norm $\left\|\left(x_{n}\right)_{n=1}^{\infty}\right\|_{\mathcal{U}}=\lim _{n \in \mathcal{U}}\left\|x_{n}\right\|$. It is then easy to see that a separable Banach space $X$ is finitely representable in $Y$ if and only if $X$ is linearly isomorphic to a subspace of the ultraproduct $Y_{\mathcal{U}}$.

A Banach space $X$ is said to be uniformly convex if given $\epsilon>0$ there exists $\delta(\epsilon)>0$ such that if $x, y \in \partial B_{X}$ then

$$
\|x+y\|>2(1-\delta) \Longrightarrow\|x-y\|<\epsilon .
$$


$X$ is uniformly smooth if given $\epsilon>0$ there exists $\delta>0$ so that if $\|x\|=1$ and $\|y\|<\delta$ then

$$
\frac{1}{2}(\|x+y\|+\|x-y\|)<1+\epsilon\|y\| .
$$

A Banach space $X$ is called super-reflexive if every space finitely representable in $X$ is reflexive, or equivalently, if every ultraproduct of $X$ is reflexive. It is a classical result of Enflo [49] and Pisier [111] that a space is super-reflexive if and only if it has an equivalent uniformly convex or (respectively, and) uniformly smooth norm.

A Banach space $X$ is called a Schur space if every weakly null sequence in $X$ converges to 0 in norm. $\ell_{1}$ is a Schur space and every infinite-dimensional closed subspace of a Schur space contains an isomorphic copy of $\ell_{1}$.

We now discuss $\mathcal{L}_{p}$-spaces. We recall that a Banach space $X$ is called a $\mathcal{L}_{p}$-space where $1 \leq p \leq \infty$ if there is a constant $\lambda>1$ so that if $E$ is a finite-dimensional subspace of $X$ there is a finite-dimensional subspace $F$ with $E \subset F \subset X$ so that $d\left(E, \ell_{p}^{m}\right) \leq \lambda$ where $m=\operatorname{dim} F$. The spaces $\ell_{p}$ and $L_{p}=L_{p}(0,1)$ provide the fundamental examples of $\mathcal{L}_{p}$-spaces when $1 \leq p<\infty$. If $p=\infty$ then $c_{0}$ and $\mathcal{C}(K)$ spaces (spaces of continuous functions) are the fundamental examples of $\mathcal{L}_{\infty}$-spaces.

A $\mathcal{L}_{2}$-space is isomorphic to a Hilbert space. If $p \neq 2$ and $1<p<\infty$ then a separable Banach space $X$ is a $\mathcal{L}_{p}$-space if it is not isomorphic to a Hilbert space and is isomorphic to a complemented subspace of $L_{p}$. The cases $p=1, \infty$ are slightly different. A separable $\mathcal{L}_{1}$-space is isomorphic to a subspace of $L_{1}$ which need not be complemented; in fact $X$ is a separable $\mathcal{L}_{1}$-space if and only if $X^{*}$ is isomorphic to $\ell_{\infty}$. Similarly $X$ is a $\mathcal{L}_{\infty}$-space if and only if $X^{* *}$ is isomorphic to a $\mathcal{C}(K)$-space. The basic theory of $\mathcal{L}_{p}$-spaces was developed in the classic paper of Lindenstrauss and Pełczyński [96].

We also recall the notions of Rademacher type and Rademacher cotype. We let $\left(\epsilon_{j}\right)_{j=1}^{\infty}$ denote a sequence of independent Rademachers (i.e., independent random variables with $\left.\mathbb{P}\left(\epsilon_{j}=1\right)=\mathbb{P}\left(\epsilon_{j}=-1\right)=\frac{1}{2}\right)$. A Banach space $X$ is said to have (Rademacher) type $p$ where $1<p \leq 2$ if there is a constant $C$ so that if $x_{1}, \ldots, x_{n} \in X$ we have

$$
\left(\mathbb{E}\left\|\sum_{j=1}^{n} \epsilon_{j} x_{j}\right\|^{p}\right)^{1 / p} \leq C\left(\sum_{j=1}^{n}\left\|x_{j}\right\|^{p}\right)^{1 / p} .
$$

$X$ is said to have (Rademacher) cotype $q$ where $2 \leq q<\infty$ if there is a constant $C$ so that if $x_{1}, \ldots, x_{n} \in X$ we have

$$
\left(\sum_{j=1}^{n}\left\|x_{j}\right\|^{q}\right)^{1 / q} \leq C\left(\mathbb{E}\left\|\sum_{j=1}^{n} \epsilon_{j} x_{j}\right\|^{q}\right)^{1 / q} .
$$

It is well-known that the spaces $L_{p}$ and $\ell_{p}$ have type $\min (p, 2)$ and cotype $\max (p, 2)$. See [5] for example.

A Banach space with nontrivial type (i.e., type $p>1$ ) always has nontrivial cotype (i.e., cotype $q<\infty$ ) but the converse is false (take $X=\ell_{1}$ ). A super-reflexive space always has nontrivial type but the converse is false [65] and [113]. 
We next consider UMD-spaces ([30]). A Banach space is said to be (UMD) (for unconditional martingale sequences) if given $1<p<\infty$ there is a constant $C$ so that $\left(\xi_{0}, \xi_{1}, \ldots, \xi_{n}\right)$ is an $X$-valued martingale and $d \xi_{j}=\xi_{j}-\xi_{j-1}$ then

$$
\left(\mathbb{E}\left\|\sum_{j=1}^{n} \theta_{j} d \xi_{j}\right\|^{p}\right)^{1 / p} \leq C\left(\mathbb{E}\left\|\xi_{n}\right\|^{p}\right)^{1 / p}
$$

whenever $\left|\theta_{j}\right| \leq 1$ for $1 \leq j \leq n$. UMD-spaces are always super-reflexive (but the converse is false [18]) and the spaces $L_{p}$ are (UMD) if $1<p<\infty$.

A good concise reference for the basics of Banach space theory is [67] or we refer to the appendices of $[16]$.

\subsection{Homeomorphisms and isometries between Banach spaces}

In this section we consider two extreme situations. In one case we are given only minimal information about the metric structure of a Banach and in the other case we have maximal information.

How much can we determine about a Banach space $X$ if all we know is the homeomorphism class of $X$ considered as a metric space? Thus we consider the following problem:

Problem 1 (Fréchet, 1928). When are two separable Banach spaces homeomorphic as metric spaces?

This question was the subject of intensive research in the years after the Second World War and was resolved beautifully by Kadets (announced in 1965) [76]:

Theorem 1.1. Any two separable infinite-dimensional Banach spaces are homeomorphic.

A result of Anderson [8] shows that all separable locally convex Fréchet spaces are also homeomorphic. Some years later, Toruńczyk completed Kadets's result by showing that any two Banach spaces (or locally convex Fréchet spaces) of the same density character are homeomorphic [123].

This is essentially the end of the story in the study of the homeomorphic theory of Banach spaces, but there is still something else to mention. If we leave the realm of locally convex spaces things get a lot mysterious. There is a very remarkable but perhaps not so well-known result of Cauty that there is a separable F-space (complete metric linear space) which is not homeomorphic to a separable Banach space [33]! Perhaps one should contrast this result of Cauty with another more recent result. In 2001, Cauty [34] showed that every compact convex subset of an F-space (even without local convexity!) has the (Schauder) fixed point property. This problem had been open since 1930, when Schauder proved the original fixed point theorem. It does not seem to be known if an infinite-dimensional compact convex set is necessarily 
homemorphic to the Hilbert cube (for subsets of a Baanch space this is essentially a result of Keller [88]). The homeomorphic theory of non-locally convex F-spaces seems to be a very rich and interesting area for future research.

The opposite end of the spectrum is to assume that we have complete information about the metric space associated to a Banach space $X$. In this situation it was shown in 1932 by Mazur and Ulam that one can recapture the real linear structure:

Theorem 1.2 (Mazur and Ulam [100]). Let $X$ and $Y$ be real Banach spaces and suppose that $U: X \rightarrow Y$ is an isometry with $U(0)=0$. Then $U$ is linear. In particular if two real Banach spaces $X$ and $Y$ are isometric as Banach spaces then they are also linearly isometric.

This, of course, raises the issue of whether the complex version of the Mazur-Ulam Theorem is valid. This question reduces to a problem in the linear theory; precisely we are asking if two complex Banach spaces which are linearly isometric as real Banach spaces are additionally linearly isometric (or even linearly isomorphic) in the complex sense. This problem aroused some interest in the 1980's. In for every complex Banach space $X$ we have a natural conjugate space $\bar{X}$ where complex multiplication is defined using conjugates, i.e., we have a new complex multiplication $\times$ given by

$$
\lambda \times x:=\bar{\lambda} x, \quad \lambda \in \mathbb{C}, x \in X .
$$

Clearly $X$ and $\bar{X}$ are real linearly isometric, but must they be complex isometric? This question was answered by Bourgain [20] negatively. He showed the existence of a space $X$ so that $X$ and $\bar{X}$ are not even linearly isomorphic. His methods were probabilistic (see also [121]); the author gave an explicit non-probabilistic construction of such a space in [78]. This space is an example of a twisted Hilbert space, i.e., a Banach space $X$ with a closed subspace $E$ so that both $E$ and $X / E$ are isometrically Hilbert spaces. The importance of such examples to the nonlinear theory is that they show that we must confine ourselves to the category of real Banach spaces in order to have a viable theory. There is no real chance of reconstructing the complex structure of a complex Banach space from the metric structure.

\subsection{Various categories of homeomorphisms}

Let $\left(M_{1}, d_{1}\right)$ and $\left(M_{2}, d_{2}\right)$ be metric spaces and suppose $f: M_{1} \rightarrow M_{2}$ is any mapping. We define the modulus of continuity of $f$ by

$$
\omega_{f}(t)=\sup \left\{d_{2}(f(x), f(y)): d_{1}(x, y) \leq t\right\}, \quad t>0 .
$$

Note that $0 \leq \omega_{f}(t) \leq \infty$. We next recall some familiar and some perhaps unfamiliar properties that $f$ may enjoy.

We say that $f$ is Lipschitz with Lipschitz constant $K$ if

$$
\omega_{f}(t) \leq K t, \quad 0<t<\infty .
$$


In this case we denote

$$
\operatorname{Lip}(f)=\inf \left\{\omega_{f}(t) / t: t>0\right\} .
$$

We say that $f$ is uniformly continuous if

$$
\lim _{t \rightarrow 0} \omega_{f}(t)=0
$$

On the other hand we say that $f$ is coarsely continuous if

$$
\omega_{f}(t)<\infty, \quad 0<t<\infty .
$$

Finally we say that $f$ is coarse Lipschitz if

$$
\limsup _{t \rightarrow \infty} \omega_{f}(t) / t<\infty
$$

In studying properties of metric spaces, we shall be studying a category whose objects are metric spaces but where the morphisms are from one of the four categories listed above.

Thus we may define two metric spaces $M_{1}, M_{2}$ to be Lipschitz isomorphic (or Lipschitz homeomorphic) if there is a Lipschitz bijection $f: M_{1} \rightarrow M_{2}$ such that $f^{-1}$ is also Lipschitz. Similarly we may consider uniformly isomorphic or, more usually uniformly homeomorphic spaces. We shall say that $M_{1}$ and $M_{2}$ are coarsely isomorphic spaces (respectively, Lipschitz coarsely isomorphic spaces) if we can choose a bijection $f: M_{1} \rightarrow M_{2}$ so that $f, f^{-1}$ are both coarse homeomorphisms (respectively Lipschitz coarse homeomorphisms).

The coarse category is a perhaps little strange because a coarsely continuous map need not be continuous. In this case the concept of coarse isomorphism is probably not appropriate, so we define a somewhat weaker form of equivalence in this category, which does not require the spaces to be of the same cardinality. Note that if $f: M_{1} \rightarrow M_{2}, g: M_{1} \rightarrow M_{2}$ are maps such that $f$ is coarsely continuous and

$$
\sup _{x \in M_{1}} d_{2}(f(x), g(x))<\infty .
$$

then $g$ is also coarsely continuous. It is reasonable to think that if $f, g$ satisfy (1) then they are in fact equivalent in some sense. This leads to the notion we call coarse homeomorphisms (note that we distinguish this from coarse isomorphism). We say that $M_{1}$ and $M_{2}$ are coarsely homeomorphic if there exists coarsely continuous maps $f: M_{1} \rightarrow M_{2}, g: M_{2} \rightarrow M_{1}$ so that

$$
\sup _{x \in M_{1}} d_{1}(x, g \circ f(x))<\infty, \quad \sup _{y \in M_{2}} d_{2}(y, f \circ g(y))<\infty .
$$

We can similarly define coarse Lipschitz homeomorphisms and coarse Lipschitz homeomorphic spaces. 
A Lipschitz embedding of $M_{1}$ into $M_{2}$ is a Lipschitz isomorphism of $M_{1}$ with a subset of $M_{2}$. (We then say that $M_{1}$ Lipschitz embeds into $M_{2}$.) Similarly a uniform embedding of $M_{1}$ into $M_{2}$ is a uniform homeomorphism between $M_{1}$ and a subset of $M_{2}$. We extend these notions to coarse (Lipschitz) embeddings using coarse (Lipschitz) homeomorphisms (not isomorphisms!)

Let us make a few remarks on the meaning of coarse homeomorphism. A coarse homeomorphism does not observe the fine structure of a metric space in the neighborhood of a point, since it is not continuous. It can only capture the macroscopic structure of the space where distances are large. This is in stark contrast to a uniform homeomorphism, which observes only the fine structure of the space. Thus if we consider $\mathbb{R}$ with its standard metric and define new metrics by

$$
\rho(x, y)=\min (|x-y|, 1), \quad \sigma(x, y)=|x-y|+1, \quad x \neq y,
$$

then $\mathbb{R}$ is uniformly, but not coarsely, homeomorphic to $(\mathbb{R}, \rho)$ and coarsely, but not uniformly, homeomorphic to $(\mathbb{R}, \sigma)$.

To understand the real meaning of a coarse homeomorphism, it is useful to introduce the idea of a skeleton (or net). If $(M, d)$ is a metric space we say that a subset $S$ is a skeleton of $M$ if there exist $0<a<b<\infty$ such that

$$
\inf _{\substack{s, t \in S \\ s \neq t}} d(s, t) \geq a, \quad \sup _{x \in M} d(x, S) \leq b .
$$

Then $M$ is also coarsely homeomorphic to each of its skeletons. Further $M_{1}, M_{2}$ are coarsely homeomorphic if and only if they have coarsely homeomorphic skeletons. It is also clear that $M_{1}$ and $M_{2}$ are coarse Lipschitz homeomorphic if and only if they have skeletons $S_{1}, S_{2}$ which are Lipschitz homeomorphic. In general, while any two skeletons of the same metric space $M$ are coarsely homeomorphic they are not necessarily Lipschitz homeomorphic, even if $M=\mathbb{R}^{2}$. For infinite-dimensional Banach spaces the situation is better:

Proposition 1.3 (Lindenstrauss, Matouskova, and Preiss (2000)). If $X$ is an infinitedimensional Banach space then any two skeletons of $X$ are Lipschitz isomorphic.

Let us recall that a metric space $(M, d)$ is metrically convex given $x, y \in M$ and $0<\lambda<1$ there exists $z \in M$ with $d(x, z)=\lambda d(x, y)$ and $d(y, z)=(1-\lambda) d(x, y)$. If $M$ is metrically convex and $f: M \rightarrow M_{1}$ is any mapping then we have subadditivity of the function $\omega_{f}$, i.e.,

$$
\omega_{f}(s+t) \leq \omega_{f}(s)+\omega_{f}(t) .
$$

This leads to the following easy Lemma:

Lemma 1.4. Suppose $M$ is metrically convex and $f: M \rightarrow M_{1}$ is any mapping, then the following are equivalent:

(i) $f$ is coarsely continuous. 
(ii) There exists $0<t<\infty$ so that $\omega_{f}(t)<\infty$.

(iii) $f$ is a coarse Lipschitz mapping.

In particular if $f$ is uniformly continuous then $f$ is also coarsely continuous.

Of course a Banach space is metrically convex so we get immediately:

Proposition 1.5. Let $X$ and $Y$ be infinite-dimensional Banach spaces. Then the following are equivalent:

(i) $X$ and $Y$ are coarsely homeomorphic.

(ii) $X$ and $Y$ are coarse Lipschitz homeomorphic.

(iii) Any skeleton of $X$ is Lipschitz homeomorphic to any skeleton of $Y$.

Conditions (i)-(iii) are implied by (iv):

(iv) $X$ and $Y$ are uniformly homeomorphic.

This Proposition summarizes a principle often referred to as the Lipschitz at large distances principle, which states that a uniform homeomorphism between Banach spaces is coarse Lipschitz. It is a curious apparent fact that although uniform homeomorphisms between Banach spaces have been studied for 40 years, the author is not aware of any result which distinguishes this concept from coarse homeomorphisms between Banach spaces.

There is an immediate application of this idea, due to Heinrich and Mankiewicz [62].

Proposition 1.6. Let $X$ and $Y$ be coarsely homeomorphic Banach spaces. Then for any non-principal ultrafilter $\mathcal{U}$ on $\mathbb{N}$, the ultraproducts $X_{\mathcal{U}}$ and $Y_{\mathcal{U}}$ are Lipschitz homeomorphic.

Proof. Suppose $f: X \rightarrow Y$ is a coarse Lipschitz homeomorphism. Define $F: X_{\mathcal{U}} \rightarrow Y_{\mathcal{U}}$ by

$$
F\left(\left(x_{n}\right)_{n=1}^{\infty}\right)=\left(f\left(n x_{n}\right) / n\right)_{n=1}^{\infty} .
$$

Then $F$ is a Lipschitz homeomorphism.

This Proposition gives an immediate link between coarse homeomorphisms (and uniform homeomorphisms) and problems concerning Lipschitz homeomorphisms. This explains why much of our effort is devoted to understanding Lipschitz isomorphisms between Banach spaces.

Let us observe that problems associated with coarse and uniform embeddings of one Banach space into another are quite different in nature because we do not require the image of the embedding to be metrically convex. Thus the arguments we have given to reach Proposition 1.5 break down completely and we have no special results 
for Banach spaces. If $\left(M_{1}, d_{1}\right),\left(M_{2}, d_{2}\right)$ are metric spaces and $f: M_{1} \rightarrow M_{2}$ is any mapping, let

$$
\varphi_{f}(t)=\inf \left\{d_{2}(f(x), f(y)): d(x, y) \geq t\right\}, \quad t>0 .
$$

Then $f$ is uniform embedding if $\varphi_{f}(t)>0$ for all $t>0$ and $\lim _{t \rightarrow 0} \omega_{f}(t)=0$ while $f$ is a coarse embedding if $\omega_{f}(t)<\infty$ for all $t>0$ and $\lim _{t \rightarrow \infty} \varphi_{f}(t)=\infty$.

We also note that significant problems arise in the study of uniform homeomorphisms between quasi-Banach spaces. If $X$ is a quasi-Banach space, then we can assume that for some $0<p<1, d(x, y)=\|x-y\|^{p}$ is a metric, but usually the space is not metrically convex and again there is no analogue of Proposition 1.5. Very little is known in this context: see, however, e.g., $[6,126]$.

\section{Lipschitz and uniform homeomorphisms between Banach spaces}

This section is devoted to the theory of Lipschitz isomorphic and uniformly homeomorphic Banach spaces. Much of the work is, by now, classical, but we will also stress some new developments.

\subsection{Classical differentiability results for Lipschitz maps}

Most of this section is very well described in [16]. Nevertheless it is important to understand the classical approach to Lipschitz isomorphism and embedding problems which is based on differentiability theorems. This is a powerful approach but has some distinct limitations as we shall see. The basic differentiability results date to the 1970s and were independently discovered by Christensen [37], Mankiewicz [97], and Aronszajn [10].

To understand the basic idea let us consider a Banach space $X$ and a Lipschitz map $f: X \rightarrow \mathbb{R}$. If $X$ is finite-dimensional then a classical result of Rademacher states that $f$ is differentiable except on a set of (Lebesgue) measure zero. Can we prove an analogous result in infinite-dimensions? The first problem we meet is to decide what sets of measure zero would look like in infinite dimensions, where there is no underlying invariant measure. One is, of course, tempted to think in terms of Baire category to define a notion of small set, but in finite dimensions this is not the correct idea for differentiability theorems. There are several solutions to this problem (see [16]) but we will follow the approach of Christensen [36] by considering Haar null sets.

We first restrict the Banach space $X$ to be separable. We then consider a Borel map $h: X \rightarrow[0, \infty)$, and say that $h$ is Haar-null if there is an $X$-valued random variable $\xi$ defined on some probability space $(\Omega, \mathbb{P})$ so that

$$
\mathbb{E} h(x+\xi)=0, \quad x \in X .
$$


A Borel subset $A$ of $X$ is Haar-null if $\chi_{A}$ is Haar-null. This notion reappeared in later work on dynamical systems [63]. It is important to note that, of course, not every set is Haar-null since $X$ itself is not.

Since $X$ is separable, in this definition, for any $\epsilon>0$ we can insist that $\|\xi\|<\epsilon$ a.e. In fact we may decompose $\Omega$ into countably many measurable sets $\Omega_{n}$ so that for each $n$ there exists $x_{n} \in X$ with $\left\|\xi(\omega)-x_{n}\right\|<\epsilon$ for $\omega \in \Omega_{n}$. Replace $\Omega$ by $\Omega^{\prime}=\Omega_{n}$ with $\mathbb{P}\left(\omega_{n}\right)>0$ and define the probability measure $\mathbb{P}^{\prime}(A)=\mathbb{P}(A) / \mathbb{P}\left(\Omega_{n}\right)$ on $\Omega_{n}$. Finally let $\xi^{\prime}=\xi-x_{n}$ on $\Omega^{\prime}$.

The key result we need is:

Lemma 2.1. If $\left(A_{n}\right)_{n=1}^{\infty}$ is a sequence of Haar-null sets then $A=\bigcup_{n=1}^{\infty} A_{n}$ is also Haar-null.

Proof. Let $h_{n}=\chi_{A_{n}}$. For each $n$ we may find a random variable $\xi_{n}$ with $\left\|\xi_{n}\right\|<2^{-n}$ a.e. so that

$$
\mathbb{E} h_{n}\left(\xi_{n}+x\right)=0, \quad x \in X .
$$

We may assume $\left(\xi_{n}\right)_{n=1}^{\infty}$ is an independent sequence and let $\xi=\sum_{n=1}^{\infty} \xi_{n}$. Then

$$
\mathbb{E} h_{n}(\xi+x)=0, \quad x \in X, n \in \mathbb{N} .
$$

Hence $\chi_{A} \leq \sum h_{n}$ is also Haar-null.

Now let $f: X \rightarrow Y$ be a Lipschitz map (we will mostly interested in the scalar case when $Y=\mathbb{R}$ ). If $X$ is infinite-dimensional there are two basic notions of differentiability available. The usual definition is that of Fréchet differentiability: $f$ is said to be Fréchet differentiable at $x_{0}$ if there is a (necessarily unique) bounded linear operator $T: X \rightarrow Y$ such that if $r(x)=f\left(x_{0}+x\right)-T x$ then

$$
\lim _{\|x\| \rightarrow 0} \frac{\|r(x)\|}{\|x\|}=0 .
$$

On the other hand there is a weaker notion available: $f$ is Gâteaux differentiable at $x_{0}$, if, with the same notation,

$$
\lim _{t \rightarrow 0} \frac{r(t x)}{t}=0, \quad x \in X .
$$

Here it is a useful fact that it suffices that the set of $x$ with

$$
\lim _{t \rightarrow 0} \frac{r(t x)}{t}=0
$$

is dense in $X$. In finite dimensions these concepts coincide but in infinite dimensions they can be quite different. Unfortunately in our circumstances we can only hope to find points of Gâteaux differentiability. 
Theorem 2.2. Let $X$ be a separable Banach space and let $f: X \rightarrow \mathbb{R}$ be a Lipschitz map. Then the set of points at which $f$ fails to be Gâteaux differentiable is Haar-null.

Remark. This is not the best possible result: one can replace Haar-null by Gaussiannull as described in [16].

Proof. Let $\left(V_{n}\right)_{n=1}^{\infty}$ be an increasing sequence of finite-dimensional subspaces of $X$ whose union is dense in $X$. We then consider the set $A_{n} \subset X$ of all points $x_{0}$ such that is a linear functional $v^{*}=v^{*}(n, x)$ on $F_{n}$ with

$$
\lim _{t \rightarrow 0} \frac{\left|f(x+t v)-t v^{*}(v)\right|}{t}=0, \quad v \in V_{n} .
$$

Let $B_{n}=X \backslash A_{n}$.

Now for fixed $n$ let $\left(e_{1}, \ldots, e_{m}\right)$ be a basis for $V_{n}$. Let $\left(\eta_{j}\right)_{j=1}^{m}$ be a sequence of independent random-variable, each uniformly distributed on $[0,1]$. Then if $\xi=$ $\sum_{j=1}^{m} \eta_{j} e_{j}$ we have

$$
\mathbb{E} \chi_{B_{n}}(\xi+x)=0, \quad x \in X
$$

by an application of Rademacher's theorem. Thus $B_{n}$ is a Haar-null set and so the union $\cup_{n=1}^{\infty} B_{n}$ is also Haar-null. It is not difficult to see that this is exactly the set on which $f$ is not Gâteaux differentiable.

This result can be extended to Lipschitz maps $f: X \rightarrow Y$ where $Y$ is a Banach space if we put a suitable restriction on $Y$. It is quite clear from the proof that we only need the validity of Rademacher's theorem for Lipschitz maps $g: V_{n} \rightarrow Y$. This holds when $Y$ has the (RNP) (see $\S 1.1$ ).

Theorem 2.3. Let $X$ be a separable Banach space and suppose $Y$ is a Banach space with (RNP). Let $f: X \rightarrow Y$ be a Lipschitz map. Then the set of points at which $f$ fails to be Gâteaux differentiable is Haar-null.

Now let us apply these results to Lipschitz embeddings:

Theorem 2.4 (Heinrich-Mankiewicz [62]). Let $X$ and $Y$ be separable Banach spaces and suppose there is a Lipschitz embedding of $X$ into $Y$. Assume $Y$ has the RadonNikodym property. Then $X$ can be linearly embedded into $Y$.

Proof. The proof of this result is now almost immediate. Suppose $f: X \rightarrow Y$ is a Lipschitz embedding so that $f$ satisfies an estimate

$$
c_{1}\|x-y\| \leq\|f(x)-f(y)\| \leq c_{2}\|x-y\|, \quad x, y \in X,
$$

where $0<c_{1}<c_{2}<\infty$. By the preceding theorem, there exists at least one point $a \in X$ so that $f$ is Gâteaux differentiable at $a$ with derivative $T$ say. We can then check that

$$
c_{1}\|x\| \leq\|T x\| \leq c_{2}\|x\|, \quad x \in X,
$$

so that $T$ is a linear embedding. 
There is a slightly more delicate argument that can be applied when $Y$ fails to have (RNP). This is due again to Heinrich and Mankiewicz [62]:

Theorem 2.5 (Heinrich-Mankiewicz [62]). Let $X$ and $Y$ be separable Banach spaces and suppose there is a Lipschitz embedding of $X$ into $Y$. Then $X$ can be linearly embedded into $Y^{* *}$.

Let us review what these results tell about classical spaces. If $Y$ is a Hilbert space then we deduce immediately that $X$ must also be a Hilbert space, up to isomorphism. This combined with Theorem 1.6 above gives a proof (not the original proof) of one of the earliest results of the subject, due to Enflo (1970):

Theorem 2.6 (Enflo [48]). A Banach space $X$ which is uniformly (or even coarsely) homeomorphic to a Hilbert space is linearly isomorphic to a Hilbert space.

We also observe that if $1 \leq p<\infty$ and $X$ can be Lipschitz embedded into $\ell_{p}$ then $X$ can be linearly embedded into $\ell_{p}$. (In the case $p=1, \ell_{1}$ has the (RNP).) The same results extend to the function spaces $L_{p}(0,1)$ but here in the case $p=1$ we must use Theorem 2.5 and the fact that every separable subspace of $L_{1}^{* *}$ embeds into $L_{1}$.

However these techniques give us no information about Banach spaces which Lipschitz embed into $c_{0}$. Indeed, in this, since $c_{0}$ fails (RNP), we can only use Theorem 2.5 and unfortunately $c_{0}^{* *}=\ell_{\infty}$ contains a (linear) isometric copy of every separable Banach space. In fact there is a very good reason why $c_{0}$ is a special case:

Theorem 2.7 (Aharoni (1974) [1]). Every separable metric metric space can be Lipschitz embedded into $c_{0}$.

Aharoni's theorem shows the limitations of differentiation techniques in studying Lipschitz embeddings. There is a curious sideplot here concerning the best result concerning the distortion of a Lipschitz embedding of a metric space into $c_{0}$. We define the distortion of a Lipschitz embedding $f: M \rightarrow M^{\prime}$ to be the quantity $\operatorname{Lip}(f) \operatorname{Lip}\left(f^{-1}\right)$. Aharoni showed that an embedding can be achieved with distortion $6+\epsilon$ for any $\epsilon>0$, i.e., with

$$
d(x, y) \leq\|f(x)-f(y)\| \leq(6+\epsilon) d(x, y), \quad x, y \in M .
$$

He noted that if $M=\ell_{1}$ we can establish a lower bound of 2 for the distortion. Shortly afterwards Assouad improved $6+\epsilon$ to $3+\epsilon$ [11]; later still Pelant [109] improved the constant to 3. Each of these authors actually found embeddings into the positive cone $c_{0}^{+}$and for this setting 3 is indeed the optimal constant. However in the last year, Lancien and the current author showed that 2 is the correct distortion constant for embeddings into $c_{0}$ [83].

A natural unsolved question here is:

Problem 2. If $c_{0}$ Lipschitz embeds into a Banach space $X$, does $c_{0}$ linearly embed into $X$ ? 
This Problem is motivated by the fact (from Theorem 2.7) that a Banach space $X$ is Lipschitz universal for all separable metric spaces if and only if $c_{0}$ Lipschitz embeds into $X$.

\subsection{The Lipschitz isomorphism problem, I}

In this section we consider one of the central problems of this area and make some initial observations. The problem is:

Problem 3. Let $X$ and $Y$ be separable Banach spaces which are Lipschitz isomorphic; are $X$ and $Y$ linearly isomorphic?

To understand this problem we must first explain why it is necessary to restrict to the separable case. If we allow non-separable spaces, then counterexamples have been known for some time. The first example was given by Aharoni and Lindenstrauss [2]. We will describe this example, which is based on the well-known fact that the quotient space $\ell_{\infty} / c_{0}$ contains a non-separable $c_{0}(\Gamma)$ (which in turn shows that $\ell_{\infty} / c_{0}$ cannot linearly embed into $\ell_{\infty}$ and thus $c_{0}$ is uncomplemented in $\left.\ell_{\infty}\right)$.

Let $q: \ell_{\infty} \rightarrow \ell_{\infty} / c_{0}$ be the canonical quotient map. We start from the existence of a continuum of infinite subsets $\left(A_{i}\right)_{i \in I}$ of $\mathbb{N}$ with the property that $A_{i} \cap A_{j}$ is finite when $i \neq j$. Let $\xi_{i}(k)=1$ if $k \in A_{i}$ and zero otherwise. Then the vectors $\left(q\left(\xi_{i}\right)\right)_{i \in I}$ are isometrically equivalent to the canonical basis vectors of $c_{0}(I)$. Let $E=\left[q\left(\xi_{i}\right)\right]_{i \in I}$. We will describe a Lipschitz map $f: E \rightarrow \ell_{\infty}$ with the property that $q \circ f=\operatorname{Id}_{E}$. If $x=\sum_{n=1}^{\infty} a_{n} q\left(\xi_{i_{n}}\right)$ where $a_{1} \geq a_{2} \geq \cdots \geq 0$, we let

$$
f(x)(k)= \begin{cases}a_{1}, & k \in A_{i_{1}} \\ a_{n}, & k \in A_{i_{n}} \backslash \bigcup_{r=1}^{n-1} A_{i_{r}}, \quad n \geq 2 \\ 0, & k \notin \bigcup_{r=1}^{\infty} A_{i_{r}}\end{cases}
$$

For general $x$ (where not all the coefficients are non-negative) we split $x=x^{+}-x^{-}$, where $x^{+}, x^{-}$have disjoint supports and non-negative coefficients. Then we set $f(x)=f\left(x^{+}\right)-f\left(x^{-}\right)$. Of course, in this definition one must verify that $f$ is defined unambiguously (it is!) and that $f$ is Lipschitz (it is with constant 2).

If $x \in q^{-1}(E)$ we define $h(x)=(x-f q(x), q(x)) \in c_{0} \oplus E$ and $h$ is a Lipschitz homeomorphism from $q^{-1}(E)$ onto $c_{0} \oplus c_{0}(I)$ which is a non-separable $c_{0}(\Gamma)$-space and hence cannot be linearly isomorphic to a subspace of $c_{0}$.

This argument was later refined by Deville, Godefroy, and Zizler [39] or [40] to show that $\mathcal{C}(K)$ is Lipschitz homeomorphic to $c_{0}(\Gamma)$ provided $K$ is a compact Hausdorff space such that the $n$-derived set $K^{(n)}=\emptyset$ for some finite $n$. Unfortunately in the separable case $(\Gamma$ countable) this does not give anything significant since these conditions imply that $\mathcal{C}(K)$ is linearly isomorphic to $c_{0}$. However in the uncountable case there are examples (as shown above) when $\mathcal{C}(K)$ is not linearly isomorphic to a $c_{0}(\Gamma)$. 
We have presented this classical example in some detail to highlight its nature. Let $X$ be a Banach space and let $Z$ be a closed subspace. Denote by $Y$ the quotient space $X / Z$ and let $q: X \rightarrow X / Z$ be the quotient mapping. Suppose we can find a Lipschitz lifting of $q$, i.e., a Lipschitz map $f: Y \rightarrow X$ so that $q \circ f=\operatorname{Id}_{Y}$. Then as before the map $h: X \rightarrow Z \oplus Y$ given by $h(x)=(x-f q(x), q(x))$ is a Lipschitz homeomorphism of $X$ onto $Z \oplus Y$. Of course, if there is a linear lifting $T: Y \rightarrow X$ of $q$ then $Z$ is complemented and $X$ must be linearly isomorphic to $Z \oplus Y$. Thus the Aharoni-Lindenstrauss approach requires the existence of a quotient map which has Lipschitz lifting but no linear lifting.

Unfortunately this approach cannot work in separable Banach spaces [53]:

Theorem 2.8. Let $X$ be a separable Banach space and let $Z$ be a closed subspace. If there exists a Lipschitz lifting $f: X / Z \rightarrow X$ of the quotient map $q: X \rightarrow X / Z$ then there is also a linear lifting $T: X / Z \rightarrow X$ with $\|T\| \leq \operatorname{Lip}(f)$.

Proof. We exploit separability to find a normalized sequence $\left(y_{n}\right)_{n=1}^{\infty}$ in $Y:=X / Z$ whose linear span is dense in $Y$. Let $\left(\xi_{n}\right)_{n=1}^{\infty}$ be a sequence of independent random variables with uniform distribution on $[-1,1]$ and let

$$
g(y)=\mathbb{E}\left(f\left(y+\sum_{n=1}^{\infty} 2^{-n} \xi_{n} y_{n}\right)\right) .
$$

Then of course $\operatorname{Lip}(g) \leq \operatorname{Lip}(f)$ and $q \circ g=q \circ f=\operatorname{Id}_{Y}$. But $g$ is Gâteaux differentiable everywhere and letting $T$ be the Gâteaux derivative at the origin gives the conclusion.

This then explains why Problem 3 is open for separable spaces!

In fact this result has applications to isometric embeddings. The Mazur-Ulam Theorem (Theorem 1.2) applies only to surjective isometries. What happens if we have an isometric embedding? In 1968, this question was addressed by Figiel [51] who proved the following:

Theorem 2.9. Let $X$ and $Y$ be Banach spaces. Suppose $f: X \rightarrow Y$ is an isometric embedding with $f(0)=0$ and that $[f(X)]=Y$. Then there is a linear operator $S: Y \rightarrow X$ with $\|S\|=1$ and $S \circ f=\operatorname{Id}_{X}$.

This means precisely that $S$ is a quotient map of $Y$ onto $X$ and that $f$ is a Lipschitz lifting with $\operatorname{Lip}(f)=1$. Applying Theorem 2.8 gives:

Theorem 2.10 ([53]). Let $X$ be a separable Banach space and suppose $X$ isometrically embeds into a Banach space $Y$. Then $X$ embeds linearly isometrically into $Y$.

We will return to the question whether an isometric embedding always implies the existence of a linear embedding for nonseparable spaces. Let us mention here a very recent preprint of Dutrieux and Lancien [45] who show, among other things, 
the existence of a compact subset $K$ of $\mathcal{C}[0,1]$ such that if $K$ embeds isometrically into a Banach space $Y$ then $\mathcal{C}[0,1]$ (and hence every separable metric space) embeds isometrically into $Y$.

Separability is of course required in Theorem 2.8 and it turns out that it also necessary in Theorem 2.10. To understand this we will introduce a canonical construction of an embedding of a metric space into a Banach space. Suppose $(M, d)$ is a metric space. We will fix one point of $M$ which we call 0 ; thus in effect we consider a pointed metric space $(M, d, 0)$. The selection of a special point is simply to avoid considering seminorms and equivalence classes in what follows. If $M=X$ is already a Banach space, we make the natural choice of the origin as our point. We define the space $\operatorname{Lip}_{0}(M)$ to be the space of all real valued Lipschitz functions $f: M \rightarrow \mathbb{R}$ with $f(0)=0$. The natural norm on $\operatorname{Lip}_{0}(M)$ is given by

$$
\|f\|_{\operatorname{Lip}_{0}(M)}=\sup \left\{\frac{|f(x)-f(y)|}{d(x, y)}: x \neq y\right\} \text {. }
$$

Then $\operatorname{Lip}_{0}(M)$ is a Banach space.

In the case when $M$ is a Banach space there is an important observation due to Lindenstrauss [94]; we give a alternate proof due to Pełczyński.

Proposition 2.11. If $X$ is a Banach space then there is a norm one projection $P$ from $\operatorname{Lip}_{0}(X)$ onto its subspace $X^{*}$. Furthermore if $E$ is any linear subspace of $X$ we can define $P=P_{E}$ with the property that if $\left.f\right|_{E}$ is linear for some subspace $E$ of $X$ then $\left.P f\right|_{E}=\left.f\right|_{E}$.

Proof. Let $E$ be a subspace of $X$. Treating $E$ as a commutative group there is an invariant mean $\mathcal{M}_{E}$ on the space $\ell_{\infty}(E ; \mathbb{R})$. If $f \in \operatorname{Lip}_{0}(X)$ we define $Q_{E} f$ by

$$
Q_{E} f(x)=\mathcal{M}_{E}\left((f(x+u)-f(u))_{u \in E}\right) .
$$

Then $Q_{E}: \operatorname{Lip}_{0}(X) \rightarrow \operatorname{Lip}_{0}(X)$ is a contractive linear operator, $Q_{E} f=f$ if $f \in X^{*}$,

$$
Q_{E} f(x+u)=Q_{E} f(x)+Q_{E} f(u), \quad x \in X, u \in E
$$

and if $\left.f\right|_{E}$ is linear

$$
Q_{E} f(u)=f(u), \quad u \in E .
$$

Let $P_{E}=Q_{X} Q_{E}$ and we have the desired projection.

Now $\operatorname{Lip}_{0}(M)$ has a natural predual. For each $x \in M$ let $\delta(x) \in \operatorname{Lip}_{0}(M)^{*}$ be point evaluation $\langle f, \delta(x)\rangle=f(x)$. Then we define $\mathbb{E}(M)$ to be the closed linear span of $\{\delta(x): x \in M\}$.

The space $Æ(M)$ was first introduced by Arens and Eells [9] in 1954 although the basic idea was due to Kantorovich [86]. The terminology Arens-Eells space, $巴(M)$ is due to Weaver [125]; a different terminology was used in [53] (the Lipschitz-free space 
of $M$, denoted $\mathcal{F}(M))$. $巴(M)$ can be described as the completion of the space of all measures on $M$ of finite support under the norm

$$
\|\mu\|_{\circledast(M)}=\inf \left\{\sum_{k=1}^{n}\left|a_{k}\right| d\left(x_{k}, y_{k}\right): \quad \mu=\sum_{k=1}^{n} a_{k}\left(\delta\left(x_{k}\right)-\delta\left(y_{k}\right)\right), n \in \mathbb{N}\right\} .
$$

The space $Æ(M)$ provides a very nice framework to study Lipschitz maps. First note that the map $\delta: M \rightarrow \mathbb{E}(M)$ sending each $x$ to the point evaluation is in fact an isometric embedding. Next observe that if $f: M_{1} \rightarrow M_{2}$ is a Lipschitz map with $f(0)=0$ then there is a naturally induced bounded linear map $T_{f}: \mathbb{E}\left(M_{1}\right) \rightarrow \mathbb{E}\left(M_{2}\right)$ such that $T_{f}(\delta(x))=\delta_{f(x)}$ and $\left\|T_{f}\right\|=\operatorname{Lip}(f)$.

Notice that there is a version of the Hahn-Banach theorem for Lipschitz maps from metric spaces into $\mathbb{R}$. Precisely if $M$ is a metric space and $A \subset M$, then a Lipschitz map $f: A \rightarrow \mathbb{R}$ can be extended with preservation of Lipschitz constant. This can be done for example by the formula

$$
f(x)=\inf _{a \in A}\{f(a)+K d(x, a)\}, \quad x \in M
$$

where $K=\operatorname{Lip}(f)$. As a consequence, if $M_{1}$ is a subset of $M_{2}$ containing the base point 0 then $\mathbb{E}\left(M_{1}\right)$ can be identified naturally and isometrically as a linear subspace of $\rightleftarrows\left(M_{2}\right)$.

If $X$ is a Banach space we additionally have a bounded linear operator $\beta: Æ(X) \rightarrow X$ given by

$$
\beta(\mu)=\int x d \mu(x)
$$

when $\mu$ is a measure with finite support. Thus $\beta(\mu)$ is the barycenter of the measure $\mu$ and we call $\beta$ the barycentric map. It is clear that $\beta: \mathbb{E}(X) \rightarrow X$ has norm one and is, in fact, a quotient map. Furthermore $\beta \circ \delta=\operatorname{Id}_{X}$. Thus $\delta$ is a Lipschitz lifting of the barycentric map, and we are exactly in the situation we had earlier in the section. If we let $Z_{X}=\operatorname{ker} \beta$, then we have that $\mathbb{E}(X)$ is Lipschitz isomorphic to $Z_{X} \oplus X$.

If $X$ is separable then $\mathbb{E}(X)$ is also separable and we are in the situation of Theorem 2.8. Thus we have the following result:

Proposition 2.12. If $X$ is a separable Banach space there is a norm one linear operator $L: X \rightarrow \circledast(X)$ with $L \circ \beta=\operatorname{Id}_{X}$.

Notice that $L^{*}: \operatorname{Lip}_{0}(X) \rightarrow X^{*}$ is a projection and so this Proposition can be regarded as a strengthening of Proposition 2.11 in the separable case, since it yields a weak*-continuous projection. However in the nonseparable setting, things are very different:

Proposition 2.13 ([53]). Let $X$ be a nonseparable reflexive space. Then every weakly compact subset of $\Subset(X)$ is separable and so $X$ is not linearly isomorphic to a subspace of $\rightleftarrows(X)$. 
This Proposition holds for weakly compactly generated spaces in place of reflexive spaces. Now we have many examples of Banach spaces $X$ which can be isometrically but not isomorphically embedded into their corresponding Arens-Eells space. From this we can also make many new examples of pairs of non-isomorphic but Lipschitz homeomorphic Banach spaces. For example if $X$ is a non-separable reflexive space then $\mathbb{E}(X)$ and $Z_{X} \oplus X$ are Lipschitz isomorphic but not linearly isomorphic. We can also see immediately that separability is required in Theorem 2.10.

Another example is also given in [53], and this has a quite simple proof:

Proposition 2.14. $\ell_{\infty}$ is not isomorphic to a subspace of $\mathbb{E}\left(\ell_{\infty}\right)$.

Proof. This is fairly easy to prove. In fact as shown in [53], since $\ell_{\infty}$ is injective, if it

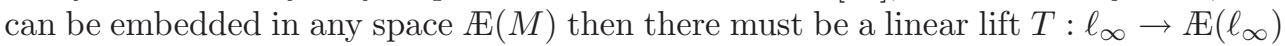
of the map $\beta$. But then $T\left(c_{0}\right)$ is contained in some $\mathbb{E}(X) \subset \mathbb{E}\left(\ell_{\infty}\right)$ where $X$ is isometric to a $\mathcal{C}(K)$-space for some compact metric space $K$. Now $X$ is a Lipschitz retract of $\ell_{\infty}$, so that there is a Lipschitz map $r: \ell_{\infty} \rightarrow X$ with $r(x)=x$ for $x \in X$ (see [94] or [16]). Hence $\Subset(X)$ is complemented in $\Subset\left(\ell_{\infty}\right)$ by some projection $P$. But then, since $\mathbb{E}(X)$ is separable $P T: \ell_{\infty} \rightarrow \mathbb{E}(X)$ is weakly compact [59] and its restriction to $c_{0}$ is compact, which gives a contradiction since $P T=T$ on $c_{0}$.

Notice the connection with Problem 2. The Banach space $\mathbb{E}\left(\ell_{\infty}\right)$ shows that this problem has a negative solution if we replace $c_{0}$ by $\ell_{\infty}$.

\subsection{The Lipschitz isomorphism problem, II}

In the previous section we saw that Problem 3 is only open for separable Banach spaces, and in this case we can attempt to use the differentiability results of $\S 2.1$. The problem is how to use the additional information that $f: X \rightarrow Y$ is a Lipschitz isomorphism. If we assume that $X$ and $Y$ are reflexive (or, more generally, have the $(\mathrm{RNP}))$ we can at least use Theorem 2.2 to find a point $x_{0} \in X$ so that $f$ has a Gâteaux derivative $S: X \rightarrow Y$ at $x_{0}$. Similarly we can apply the same result to find a point $y_{0} \in Y$ where $f^{-1}$ has a Gâteaux derivative $T: Y \rightarrow X$ at $y_{0}$. If $X$ and $Y$ where finite-dimensional we could arrange to have $y_{0}=f\left(x_{0}\right)$ and deduce by the Chain Rule that $S T=\operatorname{Id}_{Y}$ and $T S=\operatorname{Id}_{X}$. But in infinite-dimensions this is no longer possible. There are two problems. First, the notion of Gâteaux differentiability is not strong enough to give a Chain Rule. The second is that there is no guarantee that we can ensure that $y_{0}=f\left(x_{0}\right)$. The point is that if $E$ is the set of points in $X$ at which $f$ is not Gâteaux differentiable then $E$ is Haar-null but $f(E)$ need not be Haar-null (see [16, p. 149])! Thus the concept of a Haar-null set is dependent on the linear structure and not just on the metric structure.

The result of these problems is that we can obtain positive results for the Lipschitz isomorphism problem only by using some fancy footwork. We often require deep results from the linear theory to complete our arguments. Let us continue with 
results from the classic 1982 paper of Heinrich and Mankiewicz [62] which gave much of the groundwork for the present theory.

Theorem 2.15. Let $X$ and $Y$ be reflexive Banach spaces and suppose $X$ and $Y$ are Lipschitz isomorphic. Then $X$ is isomorphic to a complemented subspace of $Y$ and $Y$ is isomorphic to a complemented subspace of $X$.

Proof. Let $f: X \rightarrow Y$ be a Lipschitz homeomorphism, and let $g=f^{-1}$. We can assume by translations that $f$ is Gâteaux differentiable at 0 and that $f(0)=0$. Let $S: X \rightarrow Y$ be the Gâteaux derivative of $f$ at 0 ; then $S$ is a linear isomorphism onto a closed subspace of $Y$.

Now let $\mathcal{U}$ be a non-principal ultrafilter on $\mathbb{N}$ and define a Lipschitz function $h: Y \rightarrow X$ by

$$
h(y)=\lim _{n \in \mathcal{U}} n g(y / n)
$$

where the limit is taken in the weak topology on $X$; since $X$ is reflexive it is easy to see that $h$ is well-defined and Lipschitz. If $x \in X$ we may check that $h(S x)=x$. We use $h$ to induce a linear operator $V: X^{*} \rightarrow \operatorname{Lip}_{0}(Y)$ by setting $V x^{*}=x^{*} \circ h$. Next use Proposition 2.11 to define $T: X^{*} \rightarrow Y^{*}$ by $T=P V$ where $P: \operatorname{Lip}_{0}(Y) \rightarrow Y^{*}$ is the bounded projection constructed in the Proposition. Then observe that if $x^{*} \in X^{*}$ we have $\left.V x^{*}\right|_{S(E)}$ is linear since $V x^{*}(S x)=x^{*}(x)$. Hence $\left\langle S x, T x^{*}\right\rangle=\left\langle x, x^{*}\right\rangle$ for $x \in X$ and $x^{*} \in X^{*}$, i.e., $T^{*} S=\operatorname{Id}_{X}$. This means $X$ is isomorphic to a complemented subspace of $Y$.

This theorem brings us to a problem in the linear theory which was attracted a great deal of interest in the 1980's and 90's. Suppose $X$ is complemented in $Y$ and $Y$ is complemented in $X$ : is it true that $X$ and $Y$ must be linearly isomorphic? This was known as the Schroeder-Bernstein Problem because of its analogy to the well-known Schroeder-Bernstein Theorem. It was not until 1996 that Gowers [57] showed that it is not true in general; later Gowers and Maurey [58] gave an example of a Banach space $X$ which is isomorphic to $X^{3}$ but not to $X^{2}$. On the other hand, there are situations when a positive answer can be obtained; these date back to early work of Pełczyński [110] and are known collectively as the Petczyński decomposition trick. There are two assumptions that guarantee $X$ is isomorphic to $Y$ :

- $X$ is isomorphic to $\ell_{p}(X)$ (for some $1 \leq p<\infty$ ) or $c_{0}(X)$.

- $X \approx X^{2}$ and $Y \approx Y^{2}$.

For our purposes only the first of these hypotheses is really useful and it gives us the following result:

Theorem 2.16 (Heinrich-Mankiewicz 1982). Let $X$ be one of the spaces $\ell_{p}$ or $L_{p}$ where $1<p<\infty$. If $Y$ is Lipschitz isomorphic to $X$ then $Y$ is linearly isomorphic to $X$. 
This Theorem is our first major positive result on Problem 3. Notice that we are only able to get a positive result for a very small class of separable Banach spaces. In particular, these methods do not apply to $\ell_{1}$ or $c_{0}$. The case of $c_{0}$ is not covered by our techniques because $c_{0}$ fails the Radon-Nikodym Property; we will return to this problem later. Let us discuss the case of $\ell_{1}$. In this case the techniques we have used almost work. The space $\ell_{1}$ has the Radon-Nikodym property and so if $Y$ is Lipschitz isomorphic to $\ell_{1}$ we can use Theorem 2.4 to deduce that $Y$ is linearly isomorphic to a subspace of $\ell_{1}$ (and so it also has the (RNP)). The major obstacle is in applying Theorem 2.15. Here reflexivity was used to define $h$. It turns out that we can do without reflexivity if we have some good way of taking a limit, e.g., if $X$ is a dual space (so that we can use the weak*-topology). Thus we have:

Theorem 2.17. If $X$ is Lipschitz isomorphic to $\ell_{1}$ and is a dual space then $X$ is linearly isomorphic to $\ell_{1}$.

However the following Problem is open:

Problem 4. If $X$ is Lipschitz isomorphic to $\ell_{1}$, is $X$ linearly isomorphic to $\ell_{1}$ ?

More information on this problem follows from the following theorem. We refer to $\S 1.1$ for the definition of $\mathcal{L}_{p^{-}}$-spaces.

Theorem 2.18. Let $X$ be a $\mathcal{L}_{p}$-space where $1 \leq p \leq \infty$. If $Y$ is coarsely homeomorphic to $X$ (in particular if $Y$ is uniformly homeomorphic to $X$ ) then $Y$ is also a $\mathcal{L}_{p}$-space.

This Theorem has a long history. Notice first that since $\mathcal{L}_{p}$-spaces are stable under ultraproducts, Proposition 1.6 implies that one only needs to prove the Theorem under the hypothesis that $X$ and $Y$ are Lipschitz isomorphic.

For $1<p<\infty$, Theorem 2.18 was proved by Ribe [117]. In fact, for this case, if $X$ and $Y$ are separable then in this case Theorem 2.15 implies the conclusion (and with a little elementary theory, separability can be relaxed). The case $p=\infty$ is due to Heinrich and Mankiewicz [62] and required a different argument. However the case $p=1$ has remained open until this year, when it was finally resolved by Johnson, Maurey and Schechtman [72]. They gave an elegant approach which establishes all cases simultaneously, as a corollary of a factorization result.

Let us now turn to the case of $c_{0}$. Here differentiation techniques are quite useless. However, it turns out that there is a positive result:

Theorem 2.19 ([54]). If $X$ is Lipschitz isomorphic to $c_{0}$ then $X$ is linearly isomorphic to $c_{0}$.

To prove this result we need an argument first developed by Gorelik [56] to show that the spaces $\ell_{p}$ and $L_{p}$ are not uniformly homeomorphic when $2<p<\infty$. This is now known under the name of the Gorelik principle and it is a very remarkable 
and elegant application of the Brouwer fixed point theorem. It was primarily devised for studying uniform homeomorphisms but we state it here only for Lipschitz isomorphisms:

Proposition 2.20 (The Gorelik Principle). Let $X$ and $Y$ be Banach spaces and let $f: X \rightarrow Y$ be a Lipschitz isomorphism such that

$$
\left\|x_{1}-x_{2}\right\| \leq\left\|f\left(x_{1}\right)-f\left(x_{2}\right)\right\|
$$

for $x_{1}, x_{2} \in X$. Suppose $x \in X$ and that $E$ is a closed subspace of $X$ of finite codimension. Suppose $\delta>0$. Then for some compact set $K \subset Y$,

$$
f(x)+\delta B_{Y} \subset K+f\left(x+10 \delta B_{E}\right) .
$$

Our statement is a variant of the original Gorelik principle from [54]. To understand the Gorelik principle let us take $x=0$ and assume $f(0)=0$. If $f$ was linear then $f(E)$ would be a subspace of $Y$ of finite codimension. The Gorelik principle asserts that some remnant of this fact remains when $f$ is nonlinear; we emphasize that a more general statement applies for uniform homeomorphisms (see [16, p. 231]).

We now turn to the application of the Gorelik principle to Theorem 2.19. In fact we need only to prove:

Theorem 2.21 ([54]). If $X$ is Lipschitz isomorphic to a subspace of $c_{0}$ then $X$ is linearly isomorphic to a subspace of $c_{0}$.

The reader should recall that any Banach space which can be Lipschitz embedded into $\ell_{p}$ when $1<p<\infty$ must be linearly isomorphic to subspace of $\ell_{p}$ (Theorem 2.4); however for Lipschitz embeddings into $c_{0}$ this is false by Theorem 2.7. So the above Theorem is a little surprising; the additional hypothesis is that $X$ is Lipschitz-isomorphic to a subspace of $c_{0}$ (not merely a subset).

Once Theorem 2.21 is proved, we can invoke ideas from the linear theory to prove Theorem 2.19. Indeed $X$ must be isomorphic to $\mathcal{L}_{\infty}$-space and a subspace of $c_{0}$ and it is a classical result of Johnson and Zippin [75] that this means $X$ is isomorphic to $c_{0}$.

We turn to the proof of Theorem 2.21. Let $f: Y \rightarrow X$ be a Lipschitz isomorphism where $Y$ is a subspace of $c_{0}$. We use $f$ to define a new equivalent (dual) norm on $X^{*}$ :

$$
\left\|x^{*}\right\|_{f}=\sup \left\{\frac{x^{*}(f(v)-f(u))}{\|v-u\|}: u, v \in Y, u \neq v\right\} .
$$

The Gorelik principle is then shown to yield the property for some $c>0$ :

$$
\lim _{n \rightarrow \infty}\left\|x^{*}+x_{n}^{*}\right\|_{f} \geq\left\|x^{*}\right\|_{f}+c \lim _{n \rightarrow \infty}\left\|x_{n}^{*}\right\|_{f}
$$

whenever $x^{*} \in X^{*}$, and $\left(x_{n}^{*}\right)_{n=1}^{\infty}$ is a weak*-null sequence in $X^{*}$ such that both limits exist. The existence of such an estimate implies that $X$ is isomorphic to a subspace of $c_{0}$; such a result was first proved in almost isometric form in [85] and then in this form in [54]; an alternative proof was given in [68].

Let us also note a recent theorem of Dutrieux, related to Theorem 2.21, [43]: 
Theorem 2.22. Suppose $X$ is a Banach space such that $X^{*}$ has the (MAP). Suppose $X$ is Lipschitz isomorphic to a quotient of $c_{0}$. Then $X$ is isomorphic to a quotient of $c_{0}$.

Note that any quotient of $c_{0}$ is already isomorphic to a subspace of $c_{0}$ [7]. It would be nice to remove the approximation assumption in this result.

To conclude this section, let us consider properties which are stable under Lipschitz isomorphism. Many of the results quoted above imply stability for certain Banach space properties (e.g. reflexivity, super-reflexivity and the Radon-Nikodym property). The following is another example of such a result. For the definition of (BAP) see §1.1.

Theorem 2.23. Let $X$ and $Y$ be Lipschitz isomorphic separable Banach spaces. If $X$ has the (BAP) then $Y$ also has the (BAP).

This Theorem is proved in [53] (it also holds for non-separable spaces). The proof

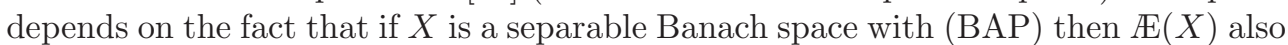
has (BAP). If $X$ and $Y$ are Lipschitz isomorphic then $Æ(X)$ and $\Subset(Y)$ are linearly isomorphic so that $\Subset(Y)$ has (BAP) and hence, since $Y$ is complemented in $Æ(Y)$ it follows that $Y$ has (BAP).

\subsection{Uniformly and coarsely homeomorphic Banach spaces}

We now consider the problem of uniform homeomorphisms between Banach spaces. We have already seen in Proposition 1.5 that if $X$ and $Y$ are uniformly homeomorphic or coarsely homeomorphic then they are coarse Lipschitz homeomorphic. Indeed, as we have already remarked, there is little in the theory of uniform homeomorphisms to distinguish between the cases of uniform and coarse homeomorphisms.

A deduction from Proposition 1.5 was that uniformly homeomorphic Banach spaces have Lipschitz-isomorphic ultraproducts (Proposition 1.6). This provides a link between the theory of uniform homeomorphisms and the theory of Lipschitz isomorphisms. In particular it gives us the following result of Ribe [116]:

Proposition 2.24. If $X$ and $Y$ are uniformly homeomorphic (or coarsely homeomorphic) Banach spaces then $X$ is finitely representable in $Y$ (and vice versa).

The immediate consequence of this Proposition is that, in general, local properties of a Banach space are preserved under uniform homeomorphism. By a local property we mean a property preserved under finite representability. For global properties this is no longer true. We now discuss examples of separable uniformly homeomorphic Banach spaces which are not linearly isomorphic. The first such example was given in 1984 by Ribe [118] and his method was extended by Aharoni and Lindenstrauss [3].

Theorem 2.25. Suppose $1 \leq p<\infty$ and $1<p_{n}<\infty$ with $\lim _{n \rightarrow \infty} p_{n}=p$ and $p_{n} \neq p$ for all $n$. Then $\ell_{2}\left(\ell_{p_{n}}\right)$ is uniformly homeomorphic, but not linearly isomorphic, to $\ell_{2}\left(\ell_{p_{n}}\right) \oplus \ell_{p}$. 
This is an intricate construction and we refer to [16, p. 244] for the details. In particular if we take $p=1$ but each $p_{n}>1$ then we see that reflexivity is not preserved under uniform homeomorphisms. So this category is quite different from the Lipschitz category.

An alternative construction was given in [79] based on the Arens-Eells space (see $\S 2.2$ ). Fix $0<\theta<1$. If $X$ is a separable Banach space we consider $X$ with metric $d_{\theta}$ given by

$$
d_{\theta}(x, y)=\max \left(\|x-y\|^{\theta},\|x-y\|\right) .
$$

Then the barycentric map $\beta$ defines a quotient map of $\mathbb{E}\left(X, d_{\theta}\right)$ onto $X$. The map $x \rightarrow \delta(x)$ is then Hölder continuous and $\beta \delta(x)=x$. From this it follows easily that $Æ\left(X, d_{\theta}\right)$ is uniformly homeomorphic to $\operatorname{ker} \beta \oplus X$. However it is shown in [79] that $Æ\left(X, d_{\theta}\right)$ is a Schur space. Hence taking, for example, $X=c_{0}$ we have that $Æ\left(c_{0}\right)$ is Lipschitz isomorphic to $\operatorname{ker} \beta \oplus c_{0}$ :

Theorem 2.26. There is a separable Schur space $Y$ which is uniformly homeomorphic to a Banach space containing $c_{0}$.

Notice the contrast with Problem 2. There is a separable Schur space $Y$ so that every separable metric space can be uniformly embedded into $Y$.

Further examples were given by Johnson, Lindenstrauss, and Schechtman [71]. Indeed they showed the existence of spaces $X$ (variants of the Tsirelson space [32]) which are uniformly homeomorphic to exactly two distinct Banach spaces.

Let us now turn to positive results. The most natural place to start is in the study of the $\ell_{p}$-spaces and $L_{p}$-spaces.

Theorem 2.27 (Enflo, Lindenstrauss). If $1 \leq p, q<\infty$ and the spaces $L_{p}(\mu)$ and $L_{q}(\mu)$ are uniformly homeomorphic then they are either of the same finite dimension or $p=q$.

Here the case $\max (p, q)>2$ was proved in 1964 by Lindenstrauss [94] and the case $\max (p, q) \leq 2$ was done in 1969 by Enflo [46]. With the benefit of hindsight these results may be proved by using Theorem 2.18 which was proved in 1978 for $1<p<\infty$ by Ribe [117]. However, the techniques used to prove Theorem 2.27 are still of considerable importance.

After this the key question is whether $\ell_{p}$ and $L_{p}$ are uniformly homeomorphic when $1 \leq p<\infty$. This was also established piecemeal.

Theorem 2.28 (Bourgain, Enflo, Gorelik). For $1 \leq p<\infty$ with $p \neq 2$ the spaces $\ell_{p}$ and $L_{p}$ are not uniformly homeomorphic.

Here the case $p=1$ is due to Enflo (unpublished) in the 1970's; the case $1<p<2$ was established in 1987 by Bourgain [22] and the case $2<p<\infty$ was not settled until 1994 by Gorelik [56].

It is worth mentioning that the case when $p<1$ is of interest in both Theorems 2.27 and 2.28. The analogue of Theorem 2.28 was established by Weston [126]. However the following problem is still open: 
Problem 5. If $0<p, q \leq 1$ are the spaces $L_{p}(0,1)$ and $L_{q}(0,1)$ uniformly homeomorphic?

The difference in the case $p<1$ as we have already remarked, is that the standard reduction to the Lipschitz case is not possible. However the spaces $L_{p}$ are metrically convex with the metric $d_{p}(f, g)=\|f-g\|_{p}^{p}$ and so we can make a reduction to a Lipschitz problem:

Problem 6. If $0<p, q \leq 1$ are the spaces $\left(L_{p}, d_{p}\right)$ and $\left(L_{q}, d_{q}\right)$ Lipschitz homeomorphic?

Let us return to the case $p \geq 1$. In 1996, Johnson, Lindenstrauss and Schechtman [71] achieved a major breakthrough by showing that if $1<p<\infty$ the spaces $\ell_{p}$ have unique uniform structure:

Theorem 2.29 (Johnson, Lindenstrauss, and Schechtman). If $1<p<\infty$ and $X$ is uniformly homeomorphic to $\ell_{p}$ then $X$ is linearly isomorphic to $\ell_{p}$.

Notice that if $p=1$ we do not even know this theorem for Lipschitz isomorphisms!

In each of the preceding theorems (Theorems $2.27,2.28$, and 2.29) we can replace uniform homeomorphisms by coarse homeomorphisms. This will be clear after our discussion below.

We will now sketch the ideas involved in Theorem 2.29 by highlighting various techniques that were developed to distinguish the uniform (or more properly, the coarse) structure of Banach spaces. The starting point is of course Ribe's theorem 2.18 which asserts that if $1<p<\infty$ and $X$ is uniformly or coarsely homeomorphic to $\ell_{p}$ then $X$ must be a $\mathcal{L}_{p}$-space. Now it follows by appealing to the linear theory and a theorem of Johnson and Odell [73] that all we need to do is show that $X$ cannot contain an isomorphic copy of $\ell_{2}$. Now $X$ is coarse Lipschitz isomorphic to $\ell_{p}$ and so we must show there is no coarse Lipschitz embedding of $\ell_{2}$ into $\ell_{p}$ when $p \neq 2$.

This leads us to the general problem of what we can say about coarse Lipschitz maps $f: \ell_{p} \rightarrow \ell_{q}$ when $p \neq q$. At the very least we want to conclude that $f$ cannot be a coarse Lipschitz embedding. Recall from the linear theory that the cases $q<p$ and $q>p$ are quite different. If $q<p$ then every bounded linear operator $T: \ell_{p} \rightarrow \ell_{q}$ is compact (Pitt's theorem) while if $q>p$ we can only deduce that $T$ is strictly singular. This difference persists in the analysis of nonlinear maps. We will discuss four basic techniques that can be used here.

First we discuss the approximate midpoint method. This approach was developed by Enflo to show that $\ell_{1}$ and $L_{1}$ are not uniformly homeomorphic. The basic idea is simple and goes back to the proof of the Mazur-Ulam Theorem. If $(M, d)$ is a metric space and $x, y \in M$ then a metric mid-point is a point $u$ so that

$$
d(u, x)=d(u, y)=\frac{1}{2} d(x, y) .
$$


If $f:\left(M_{1}, d_{1}\right) \rightarrow\left(M_{2}, d_{2}\right)$ is a Lipschitz map with Lipschitz constant $K$ and $x, y \in M_{1}$ are such that $d_{2}(f(x), f(y))=K d_{1}(x, y)$ then $f$ maps metric midpoints of $x$ and $y$ to metric midpoints of $f(x)$ and $f(y)$. In general such points $x, y$ may not exist so we resort to the definition of an approximate midpoint:

$$
\operatorname{Mid}(x, y, \delta)=\left\{z \in X: \max \{d(x, z), d(y, z)\} \leq(1+\delta) \frac{d(x, y)}{2}\right\} .
$$

Now suppose $X, Y$ are Banach spaces and $f: X \rightarrow Y$ is a coarse Lipschitz map. Define $\operatorname{Lip}_{\infty}(f)$ as the asymptotic Lipschitz constant of $f$, i.e.,

$$
\operatorname{Lip}_{\infty}(f)=\lim _{r \rightarrow \infty} \sup _{\left\|x_{1}-x_{2}\right\|>r} \frac{\left\|f\left(x_{1}\right)-f\left(x_{2}\right)\right\|}{\left\|x_{1}-x_{2}\right\|} .
$$

Then we have:

Proposition 2.30. Let $X, Y$ be Banach spaces and suppose $f: X \rightarrow M$ is a coarse Lipschitz map. If $\operatorname{Lip}_{\infty}(f)>0$ then for any $t, \epsilon>0$ and any $0<\delta<1$ there exist $x, y \in X$ with $\|x-y\|>t$ and

$$
f(\operatorname{Mid}(x, y, \delta)) \subset \operatorname{Mid}(f(x), f(y),(1+\epsilon) \delta) .
$$

Now the key fact is that we have some fairly precise information about approximate midpoint sets in $\ell_{p}$, i.e.,

Proposition 2.31. Suppose $x, y \in \ell_{p}$ where $1 \leq p<\infty$. Let $u=\frac{1}{2}(x+y)$ and $v=\frac{1}{2}(x-y)$. Then there is a compact subset $K$ of $\ell_{p}$ and a closed subspace $E$ of finite codimension so that

$$
u+\delta^{1 / p} B_{E} \subset \operatorname{Mid}(x, y, \delta) \subset K+2 \delta^{1 / p} B_{\ell_{p}} .
$$

Given Propositions 2.30 and 2.31 it is easy to see that there is no coarse Lipschitz embedding of $\ell_{p}$ into $\ell_{q}$ when $q<p$. In particular $\ell_{2}$ cannot be coarse Lipschitz embedded into $\ell_{p}$ if $p<2$. In views of our remarks above this proves Theorem 2.29 in the case when $p<2$.

The case $p>2$ requires something different. In fact, Johnson, Lindenstrauss, and Schechtman used the Gorelik principle which we have already introduced (Proposition 2.20). However we will give an alternative approach based on a recent paper [84].

Let us fix $r \in \mathbb{N}$ and for any subset $\mathbb{A}$ of $\mathbb{N}$ let $\mathcal{G}_{r}(\mathbb{A})$ be the collection of all $r$-subsets of $\mathbb{A}$. We consider $\mathcal{G}_{r}(\mathbb{N})$ as a metric space under the metric

$$
d\left(\left(m_{1}, \ldots, m_{r}\right),\left(n_{1}, \ldots, n_{r}\right)\right)=\left|\left\{j: m_{j} \neq n_{j}\right\}\right|,
$$

when $m_{1}<m_{2}<\cdots<m_{r}$ and $n_{1}<n_{2}<\cdots<n_{r}$. The crucial and quite easy Lemma we require is 
Lemma 2.32. Let $f: \mathcal{G}_{r}(\mathbb{N}) \rightarrow \ell_{p}$ be a Lipschitz map. Then given $\epsilon>0$ there is an infinite subset $\mathbb{M}$ of $\mathbb{N}$ so that

$$
\operatorname{diam}\left(\mathcal{G}_{r}(\mathbb{M})\right)<2 r^{1 / p} \operatorname{Lip}(f)+\epsilon .
$$

This Lemma can be regarded as an asymptotic smoothness condition; it has generalizations to spaces with asymptotic smoothness [84]. It follows very easily from this Lemma that there is no coarse Lipschitz embedding of $\ell_{p}$ into $\ell_{q}$ when $q>p$ and this takes care of the other case of Theorem 2.29.

It turns out the combination of Lemma 2.32 and the approximate midpoint method gives other information about direct sums:

Proposition 2.33 ([84]). Suppose $1 \leq p<q<\infty$ : then there is a coarse Lipschitz embedding of $\ell_{r}$ into $\ell_{p} \oplus \ell_{q}$ if and only if $r=p$ or $r=q$.

Theorem 2.34. If $1<p, q<\infty$ and $p, q \neq 2$ and Banach space $X$ is uniformly (or coarsely) homeomorphic to $\ell_{p} \oplus \ell_{q}$ then $X$ is linearly isomorphic to $\ell_{p} \oplus \ell_{q}$.

This Theorem is due to [71] when $p, q<2$ or $p, q>2$ and in the case $p<2<q$ is due to [84]. The argument is an extension of the arguments for the $\ell_{p}-$ case. $X$ must be linearly isomorphic to a complemented subspace of $L_{p} \oplus L_{q}$ and $X$ cannot contain a copy of $\ell_{2}$ by Proposition 2.33. These facts combined with classical techniques in the linear theory enable us to show $X$ is isomorphic to $\ell_{p} \oplus \ell_{q}$. Theorem 2.34 extends without problem to direct sums of more than two spaces.

Problem 7. If $X$ is a Banach space which is uniformly homeomorphic to $L_{p}$ (where $1<p<\infty$ and $p \neq 2$ ) is $X$ linearly isomorphic to $L_{p}$ ?

The classification of spaces uniformly homeomorphic to $L_{p}$ remains open. Clearly if $X$ is uniformly homeomorphic to $L_{p}$ then $X$ must be a $\mathcal{L}_{p}$-space and hence a complemented subspace of $L_{p}$. The simplest $\mathcal{L}_{p}$-space after $\ell_{p}$ itself is $\ell_{p} \oplus \ell_{2}$. If $p<2$ then for any $p<r<2$ we have that $\ell_{r}$ embeds linearly into $L_{p}$ and so Proposition 2.33 already gives the case $p<2$ of:

Theorem 2.35 ([84]). If $1<p<\infty$ and $p \neq 2$ then $L_{p}$ is not uniformly (or coarsely) homeomorphic to $\ell_{p} \oplus \ell_{2}$.

The case $p>2$ is a consequence of the following Theorem, again from [84] and using again the approximate mid-point technique combined with Lemma 2.32 but in a more subtle manner:

Theorem 2.36. If $1<p<\infty$ with $p \neq 2$ then if $X$ is uniformly homeomorphic to $\ell_{p} \oplus \ell_{2}$ then $X$ cannot contain a subspace isomorphic to $\ell_{p}\left(\ell_{2}\right)$.

Unfortunately these results fall a little short of establishing that $\ell_{p} \oplus \ell_{2}$ has a unique uniform structure. This is best illustrated in the case $p>2$ where the Rosenthal 
space $X_{p}([119])$ is a $\mathcal{L}_{p}$-space which embeds into $\ell_{p} \oplus \ell_{2}$ and is not eliminated by this result. However a result result of Haydon, Odell, and Schlumprecht [60] shows that in this case Theorem 2.36 shows that $X$ must be linearly isomorphic to a subspace of $\ell_{p} \oplus \ell_{2}$.

We now turn to the case of $c_{0}$ which we have seen has a unique Lipschitz structure (Theorem 2.21).

Problem 8. If $X$ is a Banach space which is uniformly homeomorphic to $c_{0}$ is $X$ linearly isomorphic to $c_{0}$ ?

Unfortunately the techniques used to prove Theorem 2.21 fail (but only just!) to give the same result in the uniform category. In [55] these techniques were used to show that certain asymptotic smoothness properties are preserved under uniform homeomorphisms. The following theorem is the main result about uniform homeomorphisms and $c_{0}$ :

Theorem 2.37. A Banach space which is uniformly homeomorphic to a subspace of $c_{0}$ has summable Szlenk index.

Rather than define summable Szlenk index let us state an equivalent form. $X$ has summable Szlenk index if and only if for every continuous function $f:(0,1) \rightarrow(0,1)$ satisfying $\lim _{\tau \rightarrow 0} f(\tau) / \tau=0$ there is a constant $c>0$ and an equivalent norm $\|\cdot\|_{f}$ on $X$ whose dual norm satisfies the condition

$$
\liminf _{n \rightarrow \infty}\left\|x^{*}+x_{n}^{*}\right\|_{f} \geq 1+c f(\tau)
$$

whenever $\left\|x^{*}\right\|_{f}=1,\left(x_{n}^{*}\right)$ is weak ${ }^{*}$-null and $\left\|x_{n}^{*}\right\|=\tau$ for all $n$.

Comparing this result with the proof of Theorem 2.21 the reader will observe that it is our inability to take $f(\tau)=\tau$ which prevents us from completing the argument. However there is some very significant information in Theorem 2.37: for example it follows that if $X$ is uniformly homeomorphic to $c_{0}$ then $X^{*}$ is isomorphic to $\ell_{1}$. It remains open whether $\ell_{1}$ has any predual, other than $c_{0}$, with summable Szlenk index.

\section{Properties of metric spaces and extension of Lipschitz maps}

In this section we consider the Lipschitz theory of metric spaces with particular emphasis on extension problems for Lipschitz maps.

\subsection{Nonlinear type and cotype}

In the following sections we will look at how concepts from the linear theory have been modified and adjusted to define corresponding nonlinear properties of metric spaces. It has proved a very fertile source of ideas that one can try to obtain Lipschitz analogues of deep results from (linear) Banach space theory. In many cases, it seems almost miraculous that such analogues do exist! 
We first discuss the notions of type and cotype for metric spaces. The idea is that we would like to describe properties of a Banach space in purely metric terms so that they are preserved under Lipschitz maps.

In the preceding sections we have seen three basic techniques which are used to study uniform and Lipschitz homeomorphism problems. These are the Gorelik principle, the approximate mid-point technique and the asymptotic smoothness principle. There is one more very elegant idea due to Enflo [46] which also has important applications. Enflo discovered that the notion of Rademacher type (see §1.1) has a nonlinear analogue. In effect, it is possible to talk of the type of a metric space.

To understand this let us define the maps $\sigma_{j}:\{-1,1\}^{n} \rightarrow\{-1,1\}^{n}$ by $\sigma_{j}\left(\epsilon_{1}, \ldots, \epsilon_{n}\right)=\left(\epsilon_{1}, \ldots,-\epsilon_{j}, \ldots, \epsilon_{n}\right)$. Let $\sigma=\sigma_{1} \cdots \sigma_{n}$. We thus say that a metric space $(M, d)$ is of Enflo type $p$ if there is a constant $C$ such that whenever $f:\{-1,1\}^{n} \rightarrow M$ is any map we have the estimate

$$
\left(\mathbb{E} d(f \circ \sigma, f)^{p}\right)^{1 / p} \leq C\left(\sum_{j=1}^{n} \mathbb{E} d\left(f \circ \sigma_{j}, f\right)^{p}\right)^{1 / p} .
$$

If $X$ is a Banach space and we consider the function

$$
f\left(\epsilon_{1}, \ldots, \epsilon_{n}\right)=\sum_{j=1}^{n} \epsilon_{j} x_{j}
$$

it is easy to see that Enflo type $p$ implies Rademacher type $p$. The remarkable fact that Enflo discovered is:

Theorem 3.1. The Banach space $L_{p}$ has Enflo type $p$ (with constant one) if $1<p \leq 2$.

From this it follows quickly that any Banach space uniformly homeomorphic to $L_{p}$ has type $p$ and this gave a proof of Theorem 2.27 in the cases when $p, q \leq 2$.

The natural question is whether Enflo type $p$ is actually equivalent to Rademacher type $p$ for a general Banach space; this was specifically raised by Enflo in [50] and remains open. As a partial answer to this, Pisier [112] proved a Poincaré-type inequality. To see this we define "partial derivatives"' by $\partial_{j} f=f \circ \sigma_{j}-f$. We also use $\mathbb{E}^{\prime}$ for expectation with respect to an independent sequence of Rademachers $\left(\epsilon_{1}^{\prime}, \ldots, \epsilon_{n}^{\prime}\right)$.

Theorem 3.2. Let $X$ be a Banach space and suppose $f:\{-1,1\}^{n} \rightarrow X$ is any map such that $\mathbb{E} f=0$. Then

$$
\left(\mathbb{E}\|f\|^{p}\right)^{1 / p} \leq(2 e \log n)\left(\mathbb{E} \mathbb{E}^{\prime}\left\|\sum_{j=1}^{n} \epsilon_{j}^{\prime} \partial_{j} f\left(\epsilon_{1}, \ldots, \epsilon_{n}\right)\right\|^{p}\right)^{1 / p} .
$$


If $X$ has type $p$ with constant $C$ this inequality yields

$$
\left(\mathbb{E}\|f\|^{p}\right)^{1 / p} \leq(2 C e \log n)\left(\mathbb{E} \sum_{j=1}^{n}\left\|\partial_{j} f\left(\epsilon_{1}, \ldots, \epsilon_{n}\right)\right\|^{p}\right)^{1 / p},
$$

and hence for general $f$ (without the condition $\mathbb{E} f=0$ ),

$$
\left(\mathbb{E}\|f-f \circ \sigma\|^{p}\right)^{1 / p} \leq(4 C e \log n)\left(\mathbb{E} \sum_{j=1}^{n}\left\|\partial_{j} f\left(\epsilon_{1}, \ldots, \epsilon_{n}\right)\right\|^{p}\right)^{1 / p} .
$$

This does not quite give Enflo type $p$ because of the logarithmic factor. However we have

Theorem 3.3 (Pisier [112]). If $X$ has type $p>1$ then $X$ has Enflo type $q$ for any $1<q<p$.

Unfortunately the $\log n$ cannot be removed in Theorem 3.2 (see [122]). However there was a recent advance in the subject due to Naor and Schechtman in 2002:

Theorem 3.4 (Naor and Schechtman [106]). Let $X$ be a UMD Banach space. Then there is a constant $C$ so that if $f:\{-1,1\}^{n} \rightarrow X$ is any map such that $\mathbb{E} f=0$, then

$$
\left(\mathbb{E}\|f\|^{p}\right)^{1 / p} \leq C\left(\mathbb{E} \mathbb{E}^{\prime}\left\|\sum_{j=1}^{n} \epsilon_{j}^{\prime} \partial_{j} f\left(\epsilon_{1}, \ldots, \epsilon_{n}\right)\right\|^{p}\right)^{1 / p}
$$

In particular for UMD-spaces, Rademacher type $p$ is equivalent to Enflo type $p$.

The Naor-Schechtman result showed for the first time that the spaces $L_{p}$ for $p>2$ have Enflo type 2.

A variant of the Enflo approach was investigated by Bourgain, Milman, and Wolfson [23]. A metric space is said to have nonlinear type $p$ if there is a constant $C$ so that whenever $f:\{-1,1\}^{n} \rightarrow M$ is any map we have the estimate

$$
\left(\mathbb{E} d(f \circ \sigma, f)^{2}\right)^{1 / 2} \leq C n^{1 / p-1 / 2}\left(\sum_{j=1}^{n} \mathbb{E} d\left(f \circ \sigma_{j}, f\right)^{2}\right)^{1 / 2} .
$$

The relationship between this property and Enflo type is not absolutely clear; however we have:

Theorem 3.5 (Bourgain, Milman, and Wolfson [23]). If a Banach space has nonlinear type $p>1$ then it has Rademacher type $q$ for every $1<q<p$.

The results of Naor and Schechtman show that for UMD-spaces we have the following implication: 
Theorem 3.6 (Naor and Schechtman [106]). Let $X$ be a UMD Banach space. If $X$ has Rademacher type $p$ then $X$ has nonlinear type $p$.

One of the main results of [23] concerns embeddings of the Hamming cube into a metric space with bounded distortion. The Hamming cube $H_{n}$ of order $n$ is the set $\{-1,1\}^{n}$ with the metric

$$
d\left(\epsilon, \epsilon^{\prime}\right)=\sum_{j=1}^{n}\left|\epsilon_{j}-\epsilon_{j}^{\prime}\right| .
$$

Theorem 3.7. In order that a metric space $(M, d)$ fail to have any nonlinear type $p>1$ it is necessary and sufficient that there exists $K<\infty$ and a sequence of Lipschitz embeddings $f_{n}: H_{n} \rightarrow M$ satisfying $\operatorname{Lip}\left(f_{n}\right) \operatorname{Lip}\left(f_{n}^{-1}\right) \leq K$.

Thus a Banach space $X$ has non-trivial type if and only if the Hamming cubes $H_{n}$ can be Lipschitz embedded into $X$ with uniformly bounded distortion.

In particular if $X$ is a Banach space we thus have a nonlinear description of nontrivial Rademacher type.

There is a corresponding metric description of super-reflexivity, which is due to Bourgain [21]. For with this we need to define the dyadic tree $T_{n}$ of order $n . T_{n}$ is defined to be the union of the sets $\{-1,1\}^{j}$ for $1 \leq j \leq n$ with the metric

$$
d\left(\left(\epsilon_{1}, \ldots, \epsilon_{j}\right),\left(\epsilon_{1}^{\prime}, \ldots, \epsilon_{k}^{\prime}\right)\right)=j+k-2 r+2
$$

where $r$ is the first index such that $\epsilon_{r} \neq \epsilon_{r}^{\prime}$.

Theorem 3.8. A Banach space $X$ fails to be super-reflexive if and only the dyadic trees $T_{n}$ can be Lipschitz embedded into $X$ with uniformly bounded distortion.

The problem of finding a suitable nonlinear analogue for cotype was only recently solved by Mendel and Naor [104]. For this we must consider a variant of the set $\{-1,1\}^{n}$. Let $m$ be an integer and let $\Gamma_{m}$ be the set of $2 m$-th. roots of unit. Let $\omega=e^{\pi i / m}$. Consider the set $\Gamma_{m}^{n}$ which we consider as a probability space with normalized counting measure. It is also a group under multiplication (and, of course, normalized counting measure is its Haar measure). We define a random variable $\xi=\left(\xi_{1}, \ldots, \xi_{n}\right)$ with values in $\Gamma_{m}^{n}$ such that $\xi_{1}, \ldots, \xi_{n}$ are independent and

$$
\mathbb{P}\left(\xi_{j}=\omega\right)=\mathbb{P}\left(\xi_{j}=1\right)=\mathbb{P}\left(\xi_{j}=\omega^{-1}\right)=1 / 3 .
$$

Let $\sigma_{j}(\theta)=\left(\theta_{1}, \ldots,-\theta_{j}, \ldots, \theta_{n}\right)$.

A metric space $(M, d)$ is said to have metric cotype $q$ if there is a constant $C$ such that if $n \in \mathbb{N}$, there exists $m \in \mathbb{N}$ so that if $f: \Gamma_{m}^{n} \rightarrow M$ is any map, then

$$
\left(\sum_{j=1}^{n} \mathbb{E}_{\theta} d\left(f(\theta), f \circ \sigma_{j}(\theta)\right)^{q}\right)^{1 / q} \leq C m\left(\mathbb{E}_{\xi} \mathbb{E}_{\theta} d(f(\theta), f(\xi \theta))^{q}\right)^{1 / q} .
$$

This rather complicated definition works very well, however: 
Theorem 3.9 (Mendel and Naor [104]). Suppose $q \geq 2$. A Banach space has Rademacher cotype $q$ if and only if it has metric cotype $q$.

Later Mendel and Naor [103] showed that a modification of the definition of Enflo type on similar lines gives a nice equivalence with Rademacher type. A metric space $M$ is said to have scaled Enflo type $p$ if there is a constant $C$ such that if $n \in \mathbb{N}$, there exists $m \in \mathbb{N}$ so that if $f: \Gamma_{m}^{n} \rightarrow M$ is any map, then

$$
\left(\mathbb{E}_{\epsilon} \mathbb{E}_{\theta} d(f(\epsilon \theta), f(\theta))^{p}\right)^{1 / p} \leq C m\left(\sum_{j=1}^{n} \mathbb{E}_{\theta} d\left(f(\theta), f\left(\alpha_{j} \theta\right)\right)^{p}\right)^{1 / p},
$$

where $\alpha_{j}=(1, \ldots, \omega, \ldots, 1)$ (with $\omega$ in the $j$ th position).

Theorem 3.10 (Mendel and Naor, [103]). Suppose $1<p \leq 2$. A Banach space has scaled Enflo type $p$ if and only if it has type $p$.

This section, however, does not totally exhaust the subject of type and cotype in metric spaces. Yet another concept is that of Markov type and Markov cotype due to Ball [13]. We will discuss these ideas later in connection with extension problems.

\subsection{The structure of the Arens-Eells space of a metric space}

Another natural way to study the structure of a pointed metric space $M$ is to attempt to understand the Arens-Eells space $Æ(M)$ as a Banach space. To motivate our considerations note that $\mathbb{E}(\mathbb{R})$ is isometric to $L_{1}$ while $\mathbb{E}(\mathbb{N}$ ) (where $\mathbb{N}$ has the natural metric) is isometric to $\ell_{1}$. These considerations suggest that the Arens-Eells space will often behave like $\ell_{1}$. However we have seen that if $X$ is a separable Banach space then $\rightleftarrows(X)$ contains a complemented copy of $X$ and so our hopes in this direction should be limited. We also note that Bourgain [21] proved that:

Theorem 3.11. The space $\mathbb{E}\left(\ell_{1}\right)$ fails to have any non-trivial (Rademacher) cotype.

Thus (since $\ell_{1}$ has cotype two) there is no realistic hope of defining a concept of cotype for a metric space using the Arens-Eells space. More recently Naor and Schechtman [107] showed:

Theorem 3.12. If $X$ is a Banach space with $\operatorname{dim} X>1$ then $\Subset(X)$ cannot be linearly embedded into $L_{1}$.

Naor and Schechtman use the term earthmover for the metric on $\mathbb{E}(X)$; this terminology is popular in theoretical computer science.

In the other direction, Godard [52] has recently given an elegant characterization of finite metric spaces $M$ such that $\Subset(M)$ is isometric to a subspace of $L_{1}$. For this we need the definition of a metric tree. We consider a connected graph $M$ with no cycles (a tree) where each edge $e$ is assigned a postive weight $w(e)$; consider the path metric $d$ induced by the weights $w(e)$. Then $(M, d)$ is called a metric tree. Godard's theorem is then: 
Theorem 3.13. Let $M$ be a finite pointed metric space. Then $\mathbb{E}(M)$ is isometric to a subspace of $L_{1}$ if and only if $M$ isometrically embeds in a finite metric tree.

This result depends on a result of Buneman [29] which characterizes those finite metric spaces which embed into a finite metric tree via the four point condition:

$$
d(x, y)+d(z, w) \leq \max (d(x, z)+d(y, w), d(x, w)+d(y, z)), \quad x, y, z, w \in M .
$$

In particular, for any metric space, $\Subset(M)$ embeds isometrically into $L_{1}$ if and only if $Æ(F)$ embeds isometrically into $L_{1}$ for every subset $F$ of $M$ with $|F|=4$. As pointed out by Godard [52] every ultrametric space satisfies the four point condition. Here a metric $d$ is an ultrametric if

$$
d(x, y) \leq \max (d(x, z), d(y, z)), \quad x, y, z \in M .
$$

For recent related work on metric trees we also refer to [42].

The above results suggest the following problem:

Problem 9. Characterize those (pointed) metric spaces $M$ so that $Æ(M)$ isomorphically embeds into $L_{1}$.

We conclude this section by discussing some special examples where $\mathbb{E}(M)$ is actually isomorphic to $\ell_{1}$.

Suppose $(M, d)$ is a compact metric space and for $0<\theta<1$ we consider the metric $d_{\theta}(x, y)=d(x, y)^{\theta}$ on $M$. We will denote $\left(M, d_{\theta}\right)$ by $M_{\theta}$; of course, Lipschitz functions on $M_{\theta}$ are simply $\theta$-Hölder functions on $M$. In this case we can define a subspace $\operatorname{lip}_{0}\left(M_{\theta}\right)$ to be the set of functions $f \in \operatorname{Lip}_{0}\left(M_{\theta}\right)$ so that

$$
\lim _{\tau \rightarrow 0} \sup \left\{\frac{|f(y)-f(x)|}{d_{\theta}(x, y)}: 0<d_{\theta}(x, y)<\tau\right\}=0 .
$$

It then follows that (see $[79,125])$ :

Proposition 3.14. $\Subset\left(M_{\theta}\right)=\operatorname{lip}_{0}\left(M_{\theta}\right)^{*}$ and $\operatorname{lip}_{0}\left(M_{\theta}\right)$ is linearly isomorphic to a subspace of $c_{0}$.

For the special case when $M$ is a compact subset of a finite-dimensional normed space, it is a classical result of Bonic, Frampton, and Tromba [17] (corrected in [125]) that:

Theorem 3.15. If $0<\theta<1$ and $M$ is a compact subset of a finite-dimensional normed space we have that $\operatorname{lip}_{0}\left(M_{\theta}\right)$ is isomorphic to $c_{0}$ and $\Subset\left(M_{\theta}\right)$ is isomorphic to $\ell_{1}$.

Weaver [125] asked whether this result would hold for any compact metric space $M$. The current author showed that this was false (Theorem 8.3 of [79]): 
Theorem 3.16. Let $M$ be a compact convex subset of a Hilbert space containing the origin. Then $\mathbb{E}\left(M_{\theta}\right)$ is isomorphic to $\ell_{1}$ if and only if $M$ is finite-dimensional.

In fact this statement holds true for any Banach space in place of a Hilbert space if $\theta \leq \frac{1}{2}$ and for almost any Banach space in general. Probably it holds in full generality for all Banach spaces (see [79]). However, it is not clear whether these counterexamples have the property that $\Subset\left(M_{\theta}\right)$ embeds into $\ell_{1}$ (or equivalently) that $\operatorname{lip}_{0}\left(M_{\theta}\right)$ is a quotient of $c_{0}$. This, of course, relates to Problem 9.

\subsection{Extension of Lipschitz maps: absolute Lipschitz retracts}

We now turn to another topic which has seen some recent advances. We will consider questions about extension of Lipschitz maps between metric spaces or Banach spaces.

Suppose $M_{1}, M_{2}$ are metric spaces and that $G \subset M_{1}$. We write $e\left(G, M_{1}, M_{2}\right)$ for the infimum of all constants $K$ so that every Lipschitz function $f: M_{1} \rightarrow M_{3}$ has an extension $\tilde{f}: G \rightarrow M_{2}$ with $\operatorname{Lip}(\tilde{f}) \leq K \operatorname{Lip}(f)$. If no such $K$ exists we write $e\left(M_{1}, M_{2}, M_{3}\right)=\infty$.

It is easy to see that if $G \subset M$ are fixed then $e\left(G, M, M^{\prime}\right)<\infty$ for every metric space $M^{\prime}$ if and only if there is a Lipschitz retraction $r: M \rightarrow G$ if a Lipschitz map such that $r: M \rightarrow G$ with $r(a)=a$ if $a \in G$. A metric space $M$ is called an absolute Lipschitz retract (ALR) if whenever $M \subset M^{\prime}$ then there is a Lipschitz retraction of $M^{\prime}$ onto $M$. This is equivalent to the statement that $e\left(G, M_{1}, M\right)<\infty$ for every pair $\left(G, M_{1}\right)$ with $G \subset M_{1}$. In this case $M$ is said to have ALR-constant $K$ if whenever $M \subset M^{\prime}$ there is a Lipschitz retraction $r: M^{\prime} \rightarrow M$ with $\operatorname{Lip}(r) \leq K$.

It is clear that $\mathbb{R}$ is an absolute Lipschitz retract; indeed $e(G, M, \mathbb{R})=1$ for all $G, M$, as we observed in $\S 2.2$. Hence the Banach space $\ell_{\infty}(\Gamma)$ is also an absolute Lipschitz retract for any set $\Gamma$ and from this it follows that every injective Banach space is an absolute Lipschitz retract. However there are other Banach spaces which are absolute Lipschitz retracts; this was shown by Lindenstrauss in 1964.

Theorem 3.17 (Lindenstrauss [94]). The spaces $c_{0}$ and $\mathcal{C}(K)$ where $K$ is compact and metrizable are absolute Lipschitz retracts.

Lindenstrauss showed that $c_{0}$ is an absolute Lipschitz retract with ALR-constant 2, i.e., if $c_{0}$ is embedded in any metric space $M$ there is a Lipschitz retraction onto $c_{0}$ with Lipschitz constant 2 (and this is best possible). This is proved by showing that there a retraction of $\ell_{\infty}$ onto $c_{0}$ with Lipschitz constant 2 . The estimate for the constant for a general $\mathcal{C}(K)$ was larger, but recently the author showed that in this case 2 is again the correct answer:

Theorem 3.18 ([82]). If $K$ is a compact metric space then $\mathcal{C}(K)$ is an absolute Lipschitz retract with ALR-constant 2.

We mention that since $\mathcal{C}(K)$ Lipschitz embeds into $c_{0}$ (Theorem 2.7) and is an absolute Lipschitz retract we get that the corresponding Arens-Eells space $\mathbb{E}(\mathcal{C}(K))$ 
is isomorphic to a complemented subspace of $\mathbb{E}\left(c_{0}\right)$. Dutrieux, and Ferenczi [44] recently used this fact and some additional arguments to show:

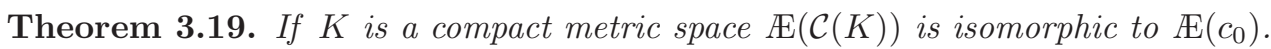

This is first known example where the Arens-Eells spaces of two non-Lipschitz isomorphic infinite-dimensional Banach spaces are known to be linearly isomorphic. For metric spaces note that Theorem 3.15 gives many examples where non-Lipschitz isomorphic metric spaces have $\ell_{1}$ as their Arens-Eells space.

Recently Lancien and Randrianantoanina [92] and the author [82] considered the following problem. For which metric spaces $M$ is it true that given any Lipschitz map $f: G \rightarrow \mathcal{C}(K)$ for some compact Hausdorff space $K$ and some $G \subset M$, there exists an extension $\tilde{f}: M \rightarrow \mathcal{C}(K)$ with $\operatorname{Lip}(\tilde{f})=\operatorname{Lip}(f)$ ? In [92] it was shown that this is never true for an infinite-dimensional Banach space.

Theorem 3.20. Let $X$ be a finite-dimensional Banach space. Then given any Lipschitz map $f: G \rightarrow \mathcal{C}(K)$ for some compact Hausdorff space $K$ and some $G \subset M$, there exists an extension $\tilde{f}: M \rightarrow \mathcal{C}(K)$ with $\operatorname{Lip}(\tilde{f})=\operatorname{Lip}(f)$ provided:

(i) $X$ is polyhedral (i.e., $X$ embeds isometrically in some $\ell_{\infty}^{N}$ ) [92].

(ii) $X$ has a Gâteaux smooth norm [82].

(iii) $\operatorname{dim} X=2[82]$.

On the other hand there is a 3-dimensional Banach space which fails this condition [82]. There is a close connection with the study of similar problems for extending linear operators into $\mathcal{C}(K)$-spaces; see [81,95] for more information.

\subsection{Extending Lipschitz maps into Banach spaces}

Let us now consider pairs $(G, M)$ such that $e(G, M, X)<\infty$ for every Banach space $X$. We fix a base point $0 \in G \subset M$. It is easy to see that this formally reduces to the statement that $e(G, M, Æ(G))<\infty$ and hence to the fact that $\mathbb{E}(G)$ is linearly complemented in $\mathbb{E}(M)$. This concept was apparently first studied by Johnson, Lindenstrauss, and Schechtman $[69,70]$.

On the other hand we may also consider the condition that there is a bounded linear extension operator $L: \operatorname{Lip}_{0}(G) \rightarrow \operatorname{Lip}_{0}(M)$. This viewpoint was recently taken by A. Brudnyi and Y. Brudnyi [28]. As noted in [28] this is equivalent to the fact that $e(G, M, X)<\infty$ for every dual space $X$. In fact it is again quite easy to see that this condition is equivalent to the fact that $Æ(G)^{* *}$ is complemented in $Æ(M)^{* *}$.

At this point we mention an important open problem (see [16, p. 183]):

Problem 10. Is every Banach space $X$ a Lipschitz retract of its bidual $X^{* *}$ ?

Of course by the discussion above every $\mathcal{C}(K)$ for $K$ compact metric is a Lipschitz retract of its bidual. If this problem has an affirmative answer then the conditions 
$e(G, M, X)<\infty$ for every Banach space $X$ and $e(G, M, X)<\infty$ for every dual Banach space are equivalent. The problem is also equivalent to asking if $\mathbb{E}(X)$ is complemented in $\Subset\left(X^{* *}\right)$ for every Banach space $X$.

If $X$ is a Banach space and $E$ is a subspace of $X$ then $E$ is said to be locally complemented in $X$ if $E^{\perp}$ is complemented in $X^{*}$. Let us make the observation that:

Proposition 3.21. If a subspace $E$ of a Banach space $X$ is a Lipschitz retract of $X$ then $E$ is locally complemented in $X$.

Proof. Let $r: X \rightarrow E$ be a Lipschitz retract. Then consider the map $\Phi: E^{*} \rightarrow$ $\operatorname{Lip}_{0}(X)$ given by $e^{*} \rightarrow e^{*} \circ r$. Let $P_{E}: \operatorname{Lip}_{0}(X) \rightarrow X^{*}$ be the projection given by Proposition 2.11, so that $\left.P_{E} f\right|_{E}=\left.f\right|_{E}$ whenever $\left.f\right|_{E} \in E^{*}$. Then $P_{E} \circ \Phi: E^{*} \rightarrow X^{*}$ is a linear extension operator and if $j: E \rightarrow X$ is the inclusion map we have that $P_{E} \circ \Phi \circ j^{*}$ is a projection whose kernel coincides with $E^{\perp}$.

Proposition 3.22. The following are equivalent:

(i) Problem 10 has an affirmative answer.

(ii) A subspace $E$ of a Banach space $X$ is locally complemented if and only if it is a Lipschitz retract of $X$.

Proof. (ii) $\Longrightarrow$ (i). The fact that $X$ is locally complemented in $X^{* *}$ is trivial since $X^{* * *}=X^{\perp} \oplus X^{*}$.

(i) $\Longrightarrow$ (ii). If $E$ is locally complemented in $X$ then $E^{* *}$ is complemented in $X^{* *}$. Hence under assumption (i) there is a Lipschitz retract of $X^{* *}$ onto $E$.

We mention also that if $X$ is Lipschitz isomorphic to $\ell_{1}$ then $X$ must be a Lipschitz retract of its bidual, and so Problem 10 has a connection with Problem 4. More precisely, we may ask:

Problem 11. If $X$ is an $\mathcal{L}_{1}$-space which is a Lipschitz retract of its bidual, must $X$ be complemented in its bidual?

Note also that if Problem 3.22 has a positive solution then every separable $\mathcal{L}_{\infty^{-}}$ space is an absolute retract.

Let us now define (following Lee and Naor [93]) the absolute extendability constant of a metric space $M$ to be the

$$
\operatorname{ae}(M)=\sup \left\{e\left(M, M^{\prime}, X\right): M \subset M^{\prime}, X \text { a Banach space }\right\} .
$$

Here ae $(M)=\infty$ if no such constant exists. Clearly ae $(M)<\infty$ if and only if $\mathbb{E}(M)$ is linearly complemented in $Æ\left(M^{\prime}\right)$ whenever $M^{\prime} \supset M$.

In this language Johnson, Lindenstrauss, and Schechtman $([69,70])$ proved the following estimates:

Theorem 3.23. (i) If $M$ is a finite metric space and $|M|=n \geq 2$ then $\operatorname{ae}(M) \leq$ $C \log n$ for some absolute constant $C$. 
(ii) If $G$ is a subset of a finite dimensional normed space $X$ with $\operatorname{dim} X=n$ then $e(G, X, Y) \leq C n$ for any Banach space $Y$ where $C$ is an absolute constant.

We remark that (ii) gives an estimate ae $(G) \leq C n^{3 / 2}$ since $X$ is an absolute retract of constant at most $n^{1 / 2}$ (a consequence of the Kadets-Snobar theorem [77]).

However, recently, Lee, and Naor [93] have proved some dramatic improvements of these results. To understand these we introduce the notion of the doubling constant of a metric space. If $M$ is a metric space the doubling constant $\lambda(M)(\leq \infty)$ is the least integer $N$ so that for every $r>0$, every ball of radius $r$ can be covered by at most $N$ balls of radius $r / 2$. This concept is a measure of finite-dimensionality - Roughly speaking $\log \lambda(M)$ can be regarded as a measure of dimension; indeed if $X$ is an $n$ dimensional normed space it is clear that $\log \lambda(X) \sim n$. It is not, however, precisely true that a metric space with finite doubling constant embeds in a finite-dimensional normed space (see [61, chapter 12]). On the other hand, Assouad [12] showed that a metric space with finite doubling constant has the property that $M_{\theta}=\left(M, d^{\theta}\right)$ Lipschitz embeds in a finite-dimensional normed space whenever $\theta<1$.

Theorem 3.24 (Lee and Naor [93]). If $M$ is a metric space with more than one point and with finite doubling constant then $\operatorname{ae}(M) \leq C \log \lambda(M)$ where $C$ is an absolute constant. In particular if $M$ is a subset of a n-dimensional normed space then ae $(M) \leq C^{\prime} n$, for absolute constant $C^{\prime}$.

This theorem gives (i) of Theorem 3.23 (since obviously $\lambda(M) \leq n$ for any $n$-point metric space) and improves (ii) of Theorem 3.23 since $\log \lambda(M) \sim n$ for any subset $M$ of an $n$-dimensional normed space. However Lee and Naor improved on Theorem 3.23 (i) as follows:

Theorem 3.25. If $M$ is a finite metric space with $|M|=n \geq 3$ then

$$
\operatorname{ae}(M) \leq C \frac{\log n}{\log \log n}
$$

for an absolute constant $C$.

The proofs of these results use techniques which go back to Whitney's work on extension of differentiable functions on subsets of $\mathbb{R}^{n}[127]$; however there is very novel ingredient of considering random partitions introduced in [93]. Let us note that the following Problem is unsolved (see [93]):

Problem 12. What is the correct asymptotic estimate for ae $(M)$ when $|M|=n$ ?

Lee and Naor point out that $\sqrt{\log n}$ is the best lower estimate known.

The next obvious step is to restrict the Banach space $X$ in the range. The most obvious case to consider is when $X$ is a Hilbert space. In this case there is an old result of Kirszbraun from 1934 [89]: 
Theorem 3.26. Let $H_{1}, H_{2}$ be Hilbert spaces and suppose $G \subset H_{1}$. If $f: G \rightarrow H_{2}$ is a Lipschitz map then there is an extension $\tilde{f}: H_{1} \rightarrow H_{2}$ with $\operatorname{Lip}(\tilde{f})=\operatorname{Lip}(f)$. Thus e $\left(G, H_{1}, H_{2}\right)=1$.

In 1984, Marcus and Pisier proved that if $G$ is an $n$-point subset of $L_{p}$ where $1<p<2$ then $e\left(G, L_{p}, L_{2}\right) \leq C(\log n)^{1 / p-1 / 2}$ for some constant $C=C(p)$. This led Johnson and Lindenstrauss to prove:

Theorem 3.27 (Johnson and Lindenstrauss [66]). Let $M$ be any metric space and let $G$ be an n-point subset of $M$; then $e\left(G, M, L_{2}\right) \leq C \sqrt{\log n}$ for some absolute constant $C$.

The method of proof of this result is interesting. The key is to prove by probabilistic arguments a dimension-reduction theorem:

Theorem 3.28 (Johnson and Lindenstrauss [66]). Given $\epsilon>0$ there exists $c=c(\epsilon)$ with the following property. Let $G$ be an n-point subset of a Hilbert space $H$. Then there is a bounded linear map $T: H \rightarrow H$ of rank $k \leq c \log n$ such that

$$
\|T x-T y\| \leq\|x-y\| \leq(1+\epsilon)\|T x-T y\|, \quad x, y \in G .
$$

The proof of Theorem 3.27 then goes as follows. Suppose $G \subset M$ is an $n$-point set, and $f: G \rightarrow L_{2}$ is any map. Select a bounded linear map $T: L_{2} \rightarrow L_{2}$ with rank $k$ less than $C \log n$ and such that

$$
\|T x-T y\| \leq\|x-y\| \leq 2\|T x-T y\|, \quad x, y \in G .
$$

Then by Theorem 3.26 there is a Lipschitz map $h: P\left(L_{2}\right) \rightarrow L_{2}$ with $\operatorname{Lip}(h) \leq 2$ and so that $h(T x)=x$ for $x \in G$. Now $T(H)$ is an absolute Lipschitz retract with constant at most $\sqrt{k}$. Hence we can extend $T \circ f$ to a Lipschitz map $g: M \rightarrow T\left(L_{2}\right)$ with $\operatorname{Lip}(g) \leq \sqrt{k} \operatorname{Lip}(f)$. Then $h \circ g: M \rightarrow L_{2}$ is our extension.

However, the dimension reduction technique of Theorem 3.28 has proved of great importance in its own right, because of applications to theoretical computer science. A companion result is that of Bourgain [19] that every $n$-point metric space is Lipschitzisomorphic to a subset of Hilbert space (which may by Theorem 3.28 be taken of dimension $C^{\prime} \log n$ ) with distortion $C \log n$.

In their paper [66], Johnson and Lindenstrauss raised the question of whether Kirszbraun's theorem (Theorem 3.26) has an extension to $L_{p}$ when $p>2$. They asked if for fixed $p>2$ the quantity $e\left(G, L_{p}, L_{2}\right)$ is always finite (and hence uniformly bounded) whenever $G$ is any subset of $L_{p}$. The motivation for this question comes from a very beautiful result in the linear theory, due to Maurey [98]:

Theorem 3.29. Let $X$ be a Banach space of type 2 and let $E$ be a closed subspace. Suppose $T: E \rightarrow H$ is a bounded operator (where $H$ is a Hilbert space). Then $T$ has a bounded linear extension extension $\tilde{T}: X \rightarrow H$.

More generally if $Y$ is a Banach space of cotype 2 and $T: E \rightarrow Y$ is a bounded operator then $T$ has a bounded linear extension $\tilde{T}: X \rightarrow Y$. 
It seems almost too good to be true that such a theorem could have a nonlinear analogue. However Ball [13] introduced some remarkable ideas in an attempt to prove just such a result. He introduced concepts of Markov type 2 and Markov cotype 2 in order to provide a corresponding a nonlinear result.

Let us define the concept of Markov type 2. We require the notion of a stationary reversible Markov chain on $\{1,2, \ldots, n\}$. This is a Markov chain $\left(Z_{t}\right)_{t=0}^{\infty}$ with stationary distribution $\mathbb{P}\left(Z_{t}=i\right)=\pi_{i}$ and transition probabilities $\mathbb{P}\left(Z_{t+1}=j \mid Z_{t}=i\right)=a_{i j}$ satisfying the reversibility condition $\pi_{i} a_{i j}=\pi_{j} a_{j i}$ for all $1 \leq i, j \leq n$. A metric space $M$ is said to have Markov type 2 with constant $K$ if given any stationary reversible Markov chain on $\{1,2, \ldots, n\}$ and any map $f:\{1,2, \ldots, n\}$ we have the estimate:

$$
\left(\mathbb{E} d\left(f\left(Z_{n}\right), f\left(Z_{0}\right)\right)^{2}\right)^{1 / 2} \leq K n^{1 / 2}\left(\mathbb{E} d\left(f\left(Z_{1}\right), f\left(Z_{0}\right)\right)^{2}\right)^{1 / 2} .
$$

We will not formally define Markov cotype 2, since Ball [13] proved that this condition holds for 2-uniformly convex Banach spaces (see below).

Let us say that a Banach space $X$ is 2-uniformly convex if there is a constant $a=a(X)$ so that

$$
\frac{1}{2}\left(\|x+y\|^{2}+\|x-y\|^{2}\right) \geq\|x\|^{2}+a^{-2}\|y\|^{2}, \quad x, y \in X
$$

and is 2-uniformly smooth if there is a constant $b=b_{2}(X)$ so that

$$
\frac{1}{2}\left(\|x+y\|^{2}+\|x-y\|^{2}\right) \leq\|x\|^{2}+b^{2}\|y\|^{2}, \quad x, y \in X .
$$

The condition of being 2-uniformly convex is stronger than simply being cotype 2 ; indeed $X$ must be uniformly convex and hence super-reflexive, so that $L_{1}$ fails to be 2 -uniformly convex. Similarly being 2 -uniformly smooth is stronger than being simply type 2 . The space $L_{p}$ for $1<p \leq 2$ are 2 -uniformly convex and for $2 \leq p<\infty$ are 2-uniformly smooth.

Ball's theorem was then:

Theorem 3.30. Let $M$ be a metric space of Markov type 2 with constant $K$ and suppose $Y$ is a 2-uniformly convex Banach space. Then $e(G, M, Y) \leq 6 K a(Y)$.

Ball further showed that a Hilbert space has Markov cotype 2 and so obtained:

Theorem 3.31. If $1<p<2$ then $e\left(G, L_{2}, L_{p}\right) \leq C(p)$ for any subset $G$ of $L_{2}$.

This paper represented a fundamental advance in the subject, but the concept of Markov type 2 remained elusive and so the problem of Johnson and Lindenstrauss was still open. This problem was settled by Tsarkov in 1999 [124] by quite different techniques:

Theorem 3.32. Let $X$ be a 2-uniformly smooth Banach space. Then there is a constant $C$ so that $e\left(G, X, L_{2}\right) \leq C$ for every subset $G$ of $X$. 
The works of Ball and Tsarkov apply to essentially dual cases. Finally, however, the situation was cleaned up by Naor, Peres, Schramm, and Sheffield [105]. They showed

Theorem 3.33. Let $X$ be a 2-uniformly smooth Banach space. Then $X$ has Markov type 2 with constant $K \leq 4 b(X)$.

Referring back to Theorem 3.30 we have

Theorem 3.34. Let $X$ be 2-uniformly smooth Banach space and let $Y$ is a 2uniformly convex Banach space. Then $e(G, X, Y) \leq 24 a(Y) b(X)$ for every subset $G$ of $X$.

In particular if $1<q<2<p<\infty$ we have

$$
e\left(G, L_{p}, L_{q}\right) \leq 24 \sqrt{\frac{p-1}{q-1}}
$$

for every subset $G$ of $L_{p}$.

These are not full analogues of Maurey's theorem because we require our spaces to have stronger conditions than type 2 and cotype 2 . The following question is thus open:

Problem 13. Is there a constant $C$ such that $e\left(G, L_{2}, L_{1}\right) \leq C$ for all subsets $G$ of $L_{2}$ ?

To finish the section, let us return to the Johnson-Lindenstrauss theorem (Theorem 3.27). This can now be obtained as a Corollary of Theorem 3.34. In fact a stronger statement is true:

Theorem 3.35. [102] Let $M$ be any metric space and let $Y$ be a 2-uniformly convex space. Then for any subset $G$ of $M$ with $n \geq 2$ points, we have $e(G, M, Y) \leq$ $C a(Y) \sqrt{\log n}$, where $C$ is an absolute constant.

Proof. Suppose $f: G \rightarrow Y$ is a map with $\operatorname{Lip}(f) \leq 1$. Let $G=\left\{a_{1}, \ldots, a_{n}\right\}$ and consider the map $h: M \rightarrow \ell_{\infty}^{n}$ given by

$$
g(x)=\left(d\left(x, a_{1}\right), \ldots, d\left(x, a_{n}\right)\right) .
$$

Then $g$ is an isometry on $G$. For $2<p<\infty$, let us consider the inclusion $j_{p}: \ell_{p}^{n} \rightarrow \ell_{\infty}^{n}$. Let $\varphi: j_{p}^{-1}(g(G)) \rightarrow Y$ be defined by $\varphi(\xi)=f\left(g^{-1} j_{p} \xi\right)$. Then $\operatorname{Lip}(\varphi) \leq \operatorname{Lip}(f)=1$. Thus there is an extension $\tilde{\varphi}: \ell_{p}^{n} \rightarrow Y$ with

$$
\operatorname{Lip}(\tilde{\varphi}) \leq 24 \sqrt{p} a(Y) .
$$

Consider finally the map $\tilde{f}=\tilde{\varphi} \circ j_{p}^{-1} \circ g$. Then $\tilde{f}$ extends $f$ and

$$
\operatorname{Lip}(\tilde{f}) \leq 24 \sqrt{p} n^{1 / p} a(Y) .
$$

Finally we optimize by taking $p \sim \log n$. 


\section{Uniform and coarse embeddings}

This final short section is devoted to recent developments in the subject of coarse and uniform embeddings of metric spaces into Banach spaces.

\subsection{Uniform and coarse embeddings in $L_{p}$-spaces}

Let us now switch gear to study problems concerning coarse and uniform embeddings. There are several types of problem which are of interest here. Let $X$ and $Y$ be Banach spaces. We are interested in these general questions:

(i) When does $X$ uniformly embed into $Y$ ?

(ii) When does $X$ coarsely embed into $Y$ ?

(iii) When does $B_{X}$ uniformly embed into $Y$ (and hence into $B_{Y}$ )?

(iv) When is $B_{X}$ uniformly homeomorphic to $B_{Y}$ ?

It is, of course, possible to have an embedding $f$ of $X$ into $Y$ which is simultaneously a uniform embedding and a coarse embedding; in this case we will say that $f$ is a strong uniform embedding. Notice that in these problems we can no longer reduce to the coarse Lipschitz case and use the Lipschitz theory. The techniques available to us are now much more restricted.

Let us consider first the classical $L_{p}$-spaces. It is well-known that $L_{q}$ is linearly isomorphic to a subspace of $L_{p}$ if and only if $p \leq q \leq 2$ (see, e.g., [5]). In view of this the following result is quite surprising:

Theorem 4.1. There is a strong uniform embedding of $L_{q}$ into $L_{p}$ if $q \leq p$. In fact there is a map $\varphi: L_{q} \rightarrow L_{p}$ such that $\|\varphi(f)-\varphi(g)\|_{p}=\|f-g\|_{q}^{q / p}$.

This result goes back to a classic paper of Bretagnolle, Dacunha-Castelle, and Krivine [25] or [24] in the case $p=2$. The general case was recently observed by Mendel and Naor [101] who gave a simple explicit embedding. It is enough, for $q<p$, to embed $L_{q}(\mathbb{R})$ into the complex space $L_{p}(\mathbb{R} \times \mathbb{R} ; \mathbb{C})$, since the complex space $L_{p}$ embeds isometrically as a real space into real $L_{p}$. We then define

$$
\varphi(f)(s, t)=a \frac{1-e^{2 i t f(s)}}{|t|^{(q+1) / p}}
$$

where

$$
a^{-p}=\int_{-\infty}^{\infty} \frac{(1-\cos t)^{p / 2}}{t^{q+1}} d t
$$


A routine calculation gives

$$
\begin{aligned}
\|\varphi(f)-\varphi(g)\|_{p}^{p} & =a^{-p} \int_{-\infty}^{\infty} \int_{-\infty}^{\infty} \frac{\left(1-\cos (t(f(s)-g(s)))^{p / 2}\right.}{|t|^{q+1}} d t d s \\
& =\int_{-\infty}^{\infty}|f(s)-g(s)|^{q} d s .
\end{aligned}
$$

The approach of Bretagnolle et al. was via an old result of Schoenberg [120]. If $K$ is any set we say that a map $f: K \times K \rightarrow \mathbb{C}$ is positive definite if

$$
\sum_{j=1}^{n} \sum_{k=1}^{n} f\left(s_{j}, s_{k}\right) \xi_{j} \overline{\xi_{k}} \geq 0
$$

for all $n \in \mathbb{N}, s_{1}, \ldots, s_{n} \in K$ and $\xi_{1}, \ldots, \xi_{n} \in \mathbb{C}$.

Theorem 4.2 (Schoenberg). A metric space $(M, d)$ isometrically embeds into a Hilbert space if and only if $K_{t}(x, y)=e^{-t d(x, y)^{2}}$ defines a positive-definite kernel on $M$ whenever $t>0$.

Now the fact that for $0<p<2, e^{-|x|^{p}}$ is the Fourier transform of a probability measure on $\mathbb{R}$ (the distribution of a $p$-stable random variable leads immediately to the conclusion that $e^{-|x-y|^{p}}$ is positive definite on $\mathbb{R}$ and hence to the fact that $K_{t}(f, g)=e^{-t\|f-g\|_{p}^{p}}$ is positive definite on $L_{p}$ for all $t>0$. Theorem 4.2 completes the proof.

Recent results of Mendel and Naor [104] complete the picture:

Theorem 4.3. In order that $L_{q}$ uniformly or coarsely embeds into $L_{p}$ it is necessary and sufficient that either $p \leq q \leq 2$ or $q \leq p$.

The key ingredient here is metric cotype; see $\S 3.1$. Mendel and Naor show that the following general result:

Theorem 4.4. Let $X$ be a Banach space with non-trivial type and cotype $p$. If $Y$ is a Banach space which coarsely or uniformly embeds into $X$ then $Y$ has cotype $p+\epsilon$ for every $\epsilon>0$.

If $q>2$ then $\ell_{q}$ cannot be either or coarsely embedded in a space with metric cotype $r<q$. Thus metric cotype (in contrast to the various forms of nonlinear type) is (somewhat) inherited by spaces which are uniformly or coarsely embedded.

We have not distinguished the case $p<1$ and the above results apply equally to these spaces. Let $d_{p}(f, g)=\|f-g\|_{p}^{p}$ when $0<p \leq 1$. We remark that it is not known if the metric spaces $\left(L_{p}, d_{p}\right)$ and $\left(L_{q}, d_{q}\right)$ could even be Lipschitz isomorphic when $0<q \leq p \leq 1$.

In 1985, Aharoni, Maurey, and Mityagin [4] completely classified those Banach spaces which uniformly embed into a Hilbert space; in 2006, a similar characterization was obtained for coarse embeddings by Randrianarivony [114] (see also [74]). In both proofs, positive-definite functions play a key role. We combine the statements: 
Theorem 4.5. In order that a Banach space $X$ (or even a quasi-Banach space) uniformly (or coarsely) embeds into a Hilbert space, it is necessary and sufficient that $X$ linearly embed into a space $L_{p}(\mu)$ when $p<1$.

If we consider uniform homeomorphisms between unit balls, then it has long been known that the unit balls of the $L_{p}(0,1)$-spaces and $\ell_{p}$-spaces are uniformly homeomorphic to $B_{\ell_{2}}$. This is achieved by the so-called Mazur map first used by Mazur in 1929 [99]. We define $\varphi: L_{p} \rightarrow L_{2}$ by

$$
\varphi(f)(s)=\|f\|^{1-p / 2}|f(s)|^{p / 2-1} f(s), \quad 0<s<1
$$

(with $\varphi(f(s))=0$ whenever $f(s)=0$ ). Then $\varphi$ is a uniform homeomorphism of $B_{L_{p}}$ onto $B_{L_{2}}$. In 1994, as a by-product of their work on the distortion problem Odell and Schlumprecht [108] discovered that it is possible to find an analogue of the Mazur map in a much more general setting. Their method was generalized in [35] and gives:

Theorem 4.6. Let $X$ be a separable Banach lattice with nontrivial cotype. Then $B_{X}$ is uniformly homeomorphic to $B_{\ell_{2}}$.

It was already known that the cotype assumption here is necessary by a result of Raynaud [115]: see Theorem 4.12 below.

The argument for Theorem 4.6 can also be interpreted in terms of complex interpolation of Banach spaces and this leads to some generalizations (see [38]). At the time the author noticed (see [16]) that one can prove more:

Theorem 4.7. Let $E$ be an infinite-dimensional subspace of a separable super-reflexive Banach lattice. Then $B_{E}$ is uniformly homeomorphic to $B_{\ell_{2}}$.

A key step in the argument for Theorem 4.7 is the following.

Proposition 4.8. Assume $E$ is a closed subspace of a super-reflexive Banach space and consider the quotient map $Q: X \rightarrow X / E$. Then it is possible to find a uniformly continuous map $\varphi: B_{X / E} \rightarrow X$ such that $Q \circ \varphi(y)=y$ for $y \in X / E$.

The proof of this Proposition is quite elementary, for we may assume that $X$ is uniformly convex and then the selection $\varphi(y)$ is the element $x$ of minimal norm such that $Q x=y$ works just fine. However once one has this one can deduce easily that $B_{X}$ is uniformly homeomorphic to $B_{E} \times B_{X / E}$. Theorem 4.7 then follows by some classical Pełczyński decomposition technique tricks (see [16, p. 202] for full details).

Problem 14. If $X$ is a separable super-reflexive space is it true that $B_{X}$ is uniformly homeomorphic to $B_{\ell_{2}}$ ?

Problem 15. Let $X$ be a closed subspace of $L_{1}$. Is $B_{X}$ uniformly homeomorphic to $B_{\ell_{2}}$ ? 
The question of whether one can find a uniformly continuous selection of a quotient map on the unit ball as in Proposition 4.8 seems to be of interest and was studied by the author in [79]. One can show for example that there is such a selection of a quotient map of $\ell_{1}$ onto $L_{1}$. The results are, unfortunately, a little technical to describe here. We will mention one concept that arises in this connection that seems to have some special importance. Let us say that $X$ (or more properly $B_{X}$ ) has (ucap) if there is an equi-uniformly continuous sequence of maps $f_{n}: B_{X} \rightarrow B_{X}$ such that $f_{n}\left(B_{X}\right)$ is compact for each $n$ and $\lim _{n \rightarrow \infty} f_{n}(x)=x$ for every $x \in B_{X}$. This is a nonlinear approximation property and we do not know if there is any Banach space which fails this condition! Every super-reflexive space has (ucap) (see [79]).

\subsection{Uniform and coarse embeddings of metric spaces}

In this section, we consider the question of whether a given metric space can be coarsely or uniformly embedded in a super-reflexive or reflexive space. It is appropriate here to consider external motivations for these questions. Let us suppose $(M, d)$ is a metric space. We say that $M$ is locally finite (respectively locally compact) if for every $x \in M$ and $r>0$ the set $\{y: d(x, y)<r\}$ is a finite set (respectively relatively compact). Notice that these terms are not being used in a standard way (in [80] locally compact is used in this sense, unfortunately without explanation). $M$ is said to have bounded geometry if given $r$ there exists $s=s(r)$ so that for every $x \in M$ and $r>0$ the set $\{y: d(x, y)<r\}$ has cardinality at most $s$. If $G$ is a group with a finite set of generators $F$ we can make $G$ into a graph by setting $x, y$ to be adjacent if either $x y^{-1}$ or $y x^{-1}$ is in $F$. This then induces a metric $d$ on $G$ so that $(G, d)$ has bounded geometry. The metric $d$ is independent on the choice of generators up to a Lipschitz isomorphism.

Recently Kasparov and $\mathrm{Yu}$ [87] showed that a metric space $(M, d)$ satisfies the coarse Novikov conjecture if $M$ coarsely embeds into a super-reflexive space; this improved an earlier result of $\mathrm{Yu}$ [128] who proved the same result for coarse embeddings into a Hilbert space. It would take us too far away from our subject to explain the meanings of these results, but it provides a motivation for understanding coarse embeddings of metric spaces into super-reflexive spaces.

Let us start by observing that not every separable metric space can be uniformly or coarsely embedded in a super-reflexive space. In fact Theorem 4.4 shows that any Banach space which can be uniformly or coarsely embedded in a super-reflexive space already has non-trivial cotype. However, recently V. Lafforgue has shown:

Theorem 4.9 (Lafforgue [91]). There is a metric space with bounded geometry which does not coarsely embed in a super-reflexive space.

We now turn to the question of embeddings into a reflexive Banach space (rather than a super-reflexive Banach space). In 2005, Brown and Guentner [27] showed that every metric space with bounded geometry can be coarsely embedded into a reflexive 
Banach space. However, this result can be improved significantly, as shown in a recent preprint of Baudier and Lancien [15]:

Theorem 4.10. Let $X$ be any Banach space failing to have nontrivial cotype. Then any locally finite metric space can be Lipschitz embedded into $X$.

Thus one can take for example the reflexive space $X=\ell_{2}\left(\ell_{\infty}^{n}\right)$. Baudier has recently also shown that every locally compact metric space strongly unifromly embeds into any space failing cotype [14].

On the other hand, it is not completely clear at this point whether every separable metric space coarsely embeds into a reflexive space. Of course this reduces, via Aharoni's theorem (Theorem 2.7) to asking whether $c_{0}$ can be coarsely embedded in a reflexive Banach space. The same problem for uniform embeddings had been around in Banach space theory for at least thirty years. Recall that we have seen (Theorem 2.26) that $c_{0}$ can be strongly uniformly embedded into a Schur space, while it is an open problem whether $c_{0}$ can be Lipschitz embedded into any Banach space not containing $c_{0}$.

Some results in this direction have been known for some time. We say that a metric space $(M, d)$ is stable if we have

$$
\lim _{m \rightarrow \infty} \lim _{n \rightarrow \infty} d\left(x_{m}, y_{n}\right)=\lim _{n \rightarrow \infty} \lim _{m \rightarrow \infty} d\left(x_{m}, y_{n}\right)
$$

whenever all limits exist. Stable Banach spaces were introduced by Krivine and Maurey in 1981 [90] and were exploited in the linear theory to obtain $\ell_{p}$-subspaces. The key fact is that the spaces $L_{p}$ for $1 \leq p<\infty$ are stable, and that every stable Banach space contains an almost isometric copy of some $\ell_{p}$ where $1 \leq p<\infty$. In particular $c_{0}$ cannot be renormed to be stable.

In 1983, Raynaud [115] discovered that stability was also a powerful tool in nonlinear theory. We will describe the underlying idea below. He showed that:

Theorem 4.11 (Raynaud [115]). $B_{c_{0}}$ cannot be uniformly embedded in any stable metric space. Hence $B_{c_{0}}$ cannot be uniformly embedded in $\ell_{2}$.

It follows quite simply from this result that:

Theorem 4.12. If $X$ is a Banach space failing cotype then $B_{X}$ cannot be uniformly embedded into $B_{\ell_{2}}$.

Recently the author found that Raynaud's techniques can be used to attack the problem of embedding into reflexive spaces. To explain the basic idea we introduce an infinite graph. Suppose $r \in \mathbb{N}$. We consider again the set $\mathcal{G}_{r}(\mathbb{N})$ of all $r$-subsets of $\mathbb{N}$. We declare that $\left(m_{1}, \ldots, m_{r}\right)$ and $\left(n_{1}, \ldots, n_{r}\right)$ (both written in increasing order) are adjacent if they are unequal and interlace, i.e., either $m_{1} \leq n_{1} \leq m_{2} \leq n_{2} \leq \cdots \leq n_{r}$ or $n_{1} \leq m_{1} \leq \cdots \leq m_{r}$. Then let $d_{p}$ be the least path metric. We then have 
Proposition 4.13. Let $(M, d)$ be a stable metric space. Let $f:\left(\mathbb{G}_{r}(\mathbb{N}), d_{p}\right) \rightarrow(M, d)$ be a Lipschitz map. Then given $\epsilon>0$ there exist $m_{1}<\cdots<m_{r}<n_{1}<\cdots<n_{r}$ so that

$$
d\left(f\left(m_{1}, \ldots, m_{r}\right), f\left(n_{1}, \ldots, n_{r}\right)\right) \leq \operatorname{Lip}(f)+\epsilon .
$$

It turns out that something similar happens when the range of $f$ is a reflexive Banach space [80]:

Proposition 4.14. Let $X$ be a reflexive Banach space. Let $f:\left(\mathbb{G}_{r}(\mathbb{N}), d_{p}\right) \rightarrow M$ be a Lipschitz map. Then given $\epsilon>0$ there exist $m_{1}<\cdots<m_{r}<n_{1}<\cdots<n_{r}$ so that

$$
d\left(f\left(m_{1}, \ldots, m_{r}\right), f\left(n_{1}, \ldots, n_{r}\right)\right) \leq 2 \operatorname{Lip}(f)+\epsilon .
$$

To see that $c_{0}$ behaves in a very different manner define the map

$$
f\left(n_{1}, \ldots, n_{r}\right)=\sum_{j=1}^{r} \sum_{k=1}^{n_{j}} e_{k}
$$

where $\left(e_{k}\right)_{k=1}^{\infty}$ is the canonical basis. Then $\operatorname{Lip}(f)=1$ but

$$
\left\|f\left(m_{1}, \ldots, m_{r}\right)-f\left(n_{1}, \ldots, n_{r}\right)\right\|=r
$$

whenever $m_{1}<m_{2}<\cdots<m_{r}<n_{1}<\cdots<n_{r}$. Thus we conclude:

Theorem 4.15 ([80]). c c cannot be coarsely embedded in a reflexive Banach space and $B_{c_{0}}$ cannot be uniformly embedded in a reflexive Banach space.

This argument can be taken further:

Theorem 4.16. Let $X$ be a Banach space so that $X, X^{*}, X^{* *}, \ldots$ are all separable (e.g., $X$ is quasi-reflexive). Then $c_{0}$ cannot be coarsely embedded in $X$ and $B_{c_{0}}$ cannot be uniformly embedded in $X$.

Of course $\ell_{1}$ can be strongly uniformly embedded in a reflexive space (e.g., $\left.\ell_{2}\right)$. On the other hand there are significant restrictions on non-reflexive spaces if we ask that either $X$ coarsely embeds into a reflexive space or that $B_{X}$ uniformly embeds into a reflexive space. Suppose $X$ contains a weakly Cauchy basic sequence $\left(x_{n}\right)_{n=1}^{\infty}$. Then we can consider the map

$$
f\left(n_{1}, \ldots, n_{r}\right)=x_{n_{1}}+\cdots+x_{n_{r}}
$$

and use somewhat similar arguments to get information. The results are:

Theorem 4.17. The following spaces have the property that $X$ cannot be coarsely embedded into a reflexive space and $B_{X}$ cannot be uniformly embedded into a reflexive space. 
(i) $X$ is non-reflexive space of non-trivial type.

(ii) $X$ is the James space [64].

The existence of non-reflexive spaces of type 2 was shown by James [65]; see also [113].

Problem 16. Suppose $X$ is a Banach space which coarsely embeds into a reflexive Banach space (or suppose $B_{X}$ uniformly embeds into a reflexive Banach space). Is $X$ weakly sequentially complete?

We also have a connection between reflexivity and stability:

Theorem 4.18 ([80]). Every stable metric space strongly uniformly embeds into a reflexive Banach space.

This applies to locally compact metric spaces (in the sense defined above!) but for locally finite spaces the Baudier-Lancien theorem (Theorem 4.10) is stronger.

Problem 17. Does every reflexive Banach space $X$ coarsely embed in a stable metric space? Does $B_{X}$ uniformly embed in a stable metric space when $X$ is reflexive?

Acknowledgements. We would like to thank a number of mathematicians whose comments have helped us to reduce the number of errors and improve the presentation of the paper; in particular we thank Fernando Cobos, Simon Cowell, Dan Fresen, Gilles Godefroy, Francisco Hernández, Bill Johnson, Gilles Lancien, and Mietek Mastyło for their comments.

\section{References}

[1] I. Aharoni, Every separable metric space is Lipschitz equivalent to a subset of $c_{0}^{+}$, Israel J. Math. 19 (1974), 284-291.

[2] I. Aharoni and J. Lindenstrauss, Uniform equivalence between Banach spaces, Bull. Amer. Math. Soc. 84 (1978), 281-283.

[3] - An extension of a result of Ribe, Israel J. Math. 52 (1985), 59-64.

[4] I. Aharoni, B. Maurey, and B. S. Mityagin, Uniform embeddings of metric spaces and of Banach spaces into Hilbert spaces, Israel J. Math. 52 (1985), no. 3, 251-265.

[5] F. Albiac and N. J. Kalton, Topics in Banach space theory, Graduate Texts in Mathematics, vol. 233, Springer, New York, 2006.

[6] Lipschitz structure of quasi-Banach spaces, Israel J. Math., to appear.

[7] D. E. Alspach, Quotients of $c_{0}$ are almost isometric to subspaces of $c_{0}$, Proc. Amer. Math. Soc. 76 (1979), 285-288.

[8] R. D. Anderson, Hilbert space is homeomorphic to the countable infinite product of lines, Bull. Amer. Math. Soc. 72 (1966), 515-519. 
[9] R. F. Arens and J. Eells Jr., On embedding uniform and topological spaces, Pacific J. Math. 6 (1956), 397-403.

[10] N. Aronszajn, Differentiability of Lipschitzian mappings between Banach spaces, Studia Math. 57 (1976), 147-190.

[11] P. Assouad, Remarques sur un article de Israel Aharoni sur les prolongements lipschitziens dans $c_{0}$ (Israel J. Math. 19 (1974), 284-291), Israel J. Math. 31 (1978), 97-100.

[12] _ Plongements lipschitziens dans $\mathbf{R}^{n}$, Bull. Soc. Math. France 111 (1983), 429-448 (French, with English summary).

[13] K. Ball, Markov chains, Riesz transforms and Lipschitz maps, Geom. Funct. Anal. 2 (1992), 137-172.

[14] F. Baudier, in preparation.

[15] F. Baudier and G. Lancien, Embeddings of locally finite metric spaces into Banach spaces. Proc. Amer. Math. Soc. to appear.

[16] Y. Benyamini and J. Lindenstrauss, Geometric nonlinear functional analysis. Vol. 1, American Mathematical Society Colloquium Publications, vol. 48, American Mathematical Society, Providence, RI, 2000

[17] R. Bonic, J. Frampton, and A. Tromba, $\Lambda$-manifolds, J. Functional Analysis 3 (1969), 310-320.

[18] J. Bourgain, Some remarks on Banach spaces in which martingale difference sequences are unconditional, Ark. Mat. 21 (1983), 163-168.

[19] $\longrightarrow$ On Lipschitz embedding of finite metric spaces in Hilbert space, Israel J. Math. 52 (1985), 46-52.

[20] Real isomorphic complex Banach spaces need not be complex isomorphic, Proc. Amer. Math. Soc. 96 (1986), 221-226.

[21] (1986), 222-230.

[22] - Remarks on the extension of Lipschitz maps defined on discrete sets and uniform homeomorphisms, Geometrical aspects of functional analysis (1985/86), Lecture Notes in Math., vol. 1267, Springer, Berlin, 1987, pp. 157-167.

[23] J. Bourgain, V. Milman, and H. Wolfson, On type of metric spaces, Trans. Amer. Math. Soc. 294 (1986), 295-317.

[24] J. Bretagnolle, D. Dacunha-Castelle, and J.-L. Krivine, Fonctions de type positif sur les espaces $L^{p}$, C. R. Acad. Sci. Paris 261 (1965), 2153-2156 (French).

[25] , Lois stables et espaces $L^{p}$, Ann. Inst. H. Poincaré Sect. B (N.S.) 2 (1965/1966), 231-259 (French).

[26] B. Brinkman and M. Charikar, On the impossibility of dimension reduction in $l_{1}$, J. ACM 52 (2005), 766-788 (electronic).

[27] N. Brown and E. Guentner, Uniform embeddings of bounded geometry spaces into reflexive Banach space, Proc. Amer. Math. Soc. 133 (2005), 2045-2050 (electronic).

[28] A. Brudnyi and Y. Brudnyi, Metric spaces with linear extensions preserving Lipschitz condition, Amer. J. Math. 129 (2007), 217-314.

[29] P. Buneman, A note on the metric properties of trees, J. Combinatorial Theory Ser. B 17 (1974), 48-50.

[30] D. L. Burkholder, A geometrical characterization of Banach spaces in which martingale difference sequences are unconditional, Ann. Probab. 9 (1981), 997-1011. 
[31] P. G. Casazza, Approximation properties, Handbook of the geometry of Banach spaces, Vol. I, 2001, pp. 271-316.

[32] P. G. Casazza and T. J. Shura, Tsirel'son's space, Lecture Notes in Mathematics, vol. 1363, Springer-Verlag, Berlin, 1989. With an appendix by J. Baker, O. Slotterbeck and R. Aron.

[33] R. Cauty, Un espace métrique linéaire qui n'est pas un rétracte absolu, Fund. Math. 146 (1994), 85-99 (French, with English summary).

[34] Solution du problème de point fixe de Schauder, Fund. Math. 170 (2001), 231-246 (French, with English summary).

[35] F. Chaatit, On uniform homeomorphisms of the unit spheres of certain Banach lattices, Pacific J. Math. 168 (1995), 11-31.

[36] J. P. R. Christensen, On sets of Haar measure zero in abelian Polish groups, Proceedings of the International Symposium on Partial Differential Equations and the Geometry of Normed Linear Spaces (Jerusalem, 1972), 1972, pp. 255-260 (1973).

[37] Measure theoretic zero sets in infinite dimensional spaces and applications to differentiability of Lipschitz mappings, Publ. Dép. Math. (Lyon) 10 (1973), 29-39. Actes du Deuxième Colloque d'Analyse Fonctionnelle de Bordeaux (Univ. Bordeaux, 1973), I, pp. 29-39.

[38] M. Daher, Homéomorphismes uniformes entre les sphères unité des espaces d'interpolation, Canad. Math. Bull. 38 (1995), 286-294 (French, with French summary).

[39] R. Deville, G. Godefroy, and V. E. Zizler, The three space problem for smooth partitions of unity and $C(K)$ spaces, Math. Ann. 288 (1990), 613-625.

[40] R. Deville, G. Godefroy, and V. Zizler, Smoothness and renormings in Banach spaces, Pitman Monographs and Surveys in Pure and Applied Mathematics, vol. 64, Longman Scientific \& Technical, Harlow, 1993.

[41] J. Diestel and J. J. Uhl Jr., Vector measures, American Mathematical Society, Providence, R.I., 1977.

[42] I. Doust and A. Weston, Enhanced negative type for finite metric trees, J. Funct. Anal., to appear.

[43] Y. Dutrieux, Quotients of $c_{0}$ and Lipschitz-homeomorphisms, Houston J. Math. 27 (2001), 593-599.

[44] Y. Dutrieux and V. Ferenczi, The Lipschitz free Banach spaces of $C(K)$-spaces, Proc. Amer. Math. Soc. 134 (2006), 1039-1044 (electronic).

[45] Y. Dutrieux and G. Lancien, Isometric embeddings of compact spaces into Banach spaces, preprint.

[46] P. Enflo, On the nonexistence of uniform homeomorphisms between $L_{p}$-spaces, Ark. Mat. 8 (1969), 103-105 (1969).

[47] - On a problem of Smirnov, Ark. Mat. 8 (1969), 107-109.

[48] Uniform structures and square roots in topological groups. I, II, Israel J. Math. 8 (1970), 230-252; ibid. 8 (1970), 253-272.

[49] Banach spaces which can be given an equivalent uniformly convex norm, Israel J. Math. 13 (1972), 281-288 (1973).

[50] - On infinite-dimensional topological groups, Séminaire sur la Géométrie des Espaces de Banach (1977-1978), École Polytech., Palaiseau, 1978, pp. Exp. No. 10-11, 11.

[51] T. Figiel, On nonlinear isometric embeddings of normed linear spaces, Bull. Acad. Polon. Sci. Sér. Sci. Math. Astronom. Phys. 16 (1968), 185-188 (English, with Loose Russian summary).

[52] A. Godard, Espaces Lipschitz-libres, propiété (M) et lissité asymptotique, Thesis, Université Paris VI, 2007. 
[53] G. Godefroy and N. J. Kalton, Lipschitz-free Banach spaces, Studia Math. 159 (2003), 121-141.

[54] G. Godefroy, N. J. Kalton, and G. Lancien, Subspaces of $c_{0}(\mathbf{N})$ and Lipschitz isomorphisms, Geom. Funct. Anal. 10 (2000), 798-820.

[55] , Szlenk indices and uniform homeomorphisms, Trans. Amer. Math. Soc. 353 (2001), 3895-3918 (electronic).

[56] E. Gorelik, The uniform nonequivalence of $L_{p}$ and $l_{p}$, Israel J. Math. 87 (1994), 1-8.

[57] W. T. Gowers, A solution to the Schroeder-Bernstein problem for Banach spaces, Bull. London Math. Soc. 28 (1996), 297-304.

[58] W. T. Gowers and B. Maurey, Banach spaces with small spaces of operators, Math. Ann. 307 (1997), 543-568.

[59] A. Grothendieck, Sur les applications linéaires faiblement compactes d'espaces du type $C(K)$, Canadian J. Math. 5 (1953), 129-173 (French).

[60] R. Haydon, E. Odell, and Th. Schlumprecht, Small subspaces of $L_{p}$, preprint, 2007.

[61] J. Heinonen, Lectures on analysis on metric spaces, Universitext, Springer-Verlag, New York, 2001.

[62] S. Heinrich and P. Mankiewicz, Applications of ultrapowers to the uniform and Lipschitz classification of Banach spaces, Studia Math. 73 (1982), 225-251.

[63] B. R. Hunt, T. Sauer, and J. A. Yorke, Prevalence: a translation-invariant "almost every" on infinite-dimensional spaces, Bull. Amer. Math. Soc. (N.S.) 27 (1992), 217-238.

[64] R. C. James, A non-reflexive Banach space isometric with its second conjugate space, Proc. Nat. Acad. Sci. U. S. A. 37 (1951), 174-177.

[65] _ Nonreflexive spaces of type 2, Israel J. Math. 30 (1978), 1-13.

[66] W. B. Johnson and J. Lindenstrauss, Extensions of Lipschitz mappings into a Hilbert space, Conference in modern analysis and probability (New Haven, Conn., 1982), Contemp. Math., vol. 26, Amer. Math. Soc., Providence, RI, 1984, pp. 189-206.

[67]__ Basic concepts in the geometry of Banach spaces, Handbook of the geometry of Banach spaces, Vol. I, 2001, pp. 1-84.

[68] W. B. Johnson, J. Lindenstrauss, D. Preiss, and G. Schechtman, Almost Fréchet differentiability of Lipschitz mappings between infinite-dimensional Banach spaces, Proc. London Math. Soc. (3) 84 (2002), 711-746.

[69] W. B. Johnson, J. Lindenstrauss, and G. Schechtman, Extensions of Lipschitz maps into Banach spaces, Israel seminar on geometrical aspects of functional analysis (1983/84), Tel Aviv Univ., Tel Aviv, 1984, pp. I, 15.

[70] _ Extensions of Lipschitz maps into Banach spaces, Israel J. Math. 54 (1986), 129-138.

[71] Banach spaces determined by their uniform structures, Geom. Funct. Anal. 6 (1996), $430-470$.

[72] W. B. Johnson, B. Maurey, and G. Schechtman, Non-linear factorization of linear operators, to appear.

[73] W. B. Johnson and E. Odell, Subspaces of $L_{p}$ which embed into $l_{p}$, Compositio Math. 28 (1974), 37-49.

[74] W. B. Johnson and N. L. Randrianarivony, $l_{p}(p>2)$ does not coarsely embed into a Hilbert space, Proc. Amer. Math. Soc. 134 (2006), 1045-1050 (electronic).

[75] W. B. Johnson and M. Zippin, On subspaces of quotients of $\left(\sum G_{n}\right)_{l p}$ and $\left(\sum G_{n}\right)_{c_{0}}$, Proceedings of the International Symposium on Partial Differential Equations and the Geometry of Normed Linear Spaces (Jerusalem, 1972), 1972, pp. 311-316 (1973). 
[76] M. I. Kadets, A proof of the topological equivalence of all separable infinite-dimensional Banach spaces, Funkcional. Anal. i Priložen. 1 (1967), 61-70 (Russian).

[77] M. I. Kadets and M. G. Snobar, Certain functionals on the Minkowski compactum, Mat. Zametki 10 (1971), 453-457 (Russian).

[78] N. J. Kalton, An elementary example of a Banach space not isomorphic to its complex conjugate, Canad. Math. Bull. 38 (1995), 218-222.

[79] Spaces of Lipschitz and Hölder functions and their applications, Collect. Math. $\mathbf{5 5}$ (2004), 171-217.

[80] Coarse and uniform embeddings into reflexive spaces, Quart. J. Math. (Oxford) 58 (2007), 393-414.

[81] Extension of linear operators and Lipschitz maps into $\mathcal{C}(K)$-spaces, New York J. Math. 13 (2007), 317-381.

[82] _ Extending Lipschitz maps into $\mathcal{C}(K)$ - spaces, Israel J. Math. to appear.

[83] N. J. Kalton and G. Lancien, Best constants for Lipschitz embeddings of metric spaces into $c_{0}$, to appear.

[84] N. J. Kalton and N. L. Randrianarivony, The coarse Lipschitz structure of $\ell_{p} \oplus \ell_{q}$, Math. Ann., to appear.

[85] N. J. Kalton and D. Werner, Property $(M), M$-ideals, and almost isometric structure of Banach spaces, J. Reine Angew. Math. 461 (1995), 137-178.

[86] L. V. Kantorovich, On the translocation of masses, Dokl. Akad. Nauk SSSR 37 (1942), 227-229.

[87] G. Kasparov and G. Yu, The coarse geometric Novikov conjecture and uniform convexity, Adv. Math. 206 (2006), 1-56.

[88] O-H. Keller, Die Homoiomorphie der kompakten konvexen Mengen im Hilbertschen Raum, Math. Ann. 105 (1931), 748-758 (German).

[89] M. D. Kirszbraun, Über die zusammenzieheiden und Lipschitzchen Transformationen, Fund. Math. 22 (1934), 77-108.

[90] J. L. Krivine and B. Maurey, Espaces de Banach stables, Israel J. Math. 39 (1981), 273-295 (French, with English summary).

[91] V. Lafforgue, Un renforcement de la propriété (T), to appear.

[92] G. Lancien and B. Randrianantoanina, On the extension of Hölder maps with values in spaces of continuous functions, Israel J. Math. 147 (2005), 75-92.

[93] J. R. Lee and A. Naor, Extending Lipschitz functions via random metric partitions, Invent. Math. 160 (2005), 59-95.

[94] J. Lindenstrauss, On nonlinear projections in Banach spaces, Michigan Math. J. 11 (1964), 263-287.

[95] _ Extension of compact operators, Mem. Amer. Math. Soc. No. 48 (1964), 112.

[96] J. Lindenstrauss and A. Pełczyński, Absolutely summing operators in $L_{p}$-spaces and their applications, Studia Math. 29 (1968), 275-326.

[97] P. Mankiewicz, On the differentiability of Lipschitz mappings in Fréchet spaces, Studia Math. 45 (1973), 15-29.

[98] B. Maurey, Un théorème de prolongement, C. R. Acad. Sci. Paris Sér. A 279 (1974), 329-332 (French).

[99] S. Mazur, Une remarque sur l'homéomorphie des champs fonctionnels, Studia Math. 1 (1929), $83-85$. 
[100] S. Mazur and S. Ulam, Sur les transformations isométriques d'espaces vectoriels normés, C. R. Acad. Sci. Paris 194 (1932), 946-948.

[101] M. Mendel and A. Naor, Euclidean quotients of finite metric spaces, Adv. Math. 189 (2004), $451-494$.

[102] Some applications of Ball's extension theorem, Proc. Amer. Math. Soc. 134 (2006), 2577-2584 (electronic).

[103] Scaled Enflo type is equivalent to Rademacher type, Bull. Lond. Math. Soc. 39 (2007), 493-498.

[104] _ Metric cotype, Ann. Math., to appear.

[105] A. Naor, Y. Peres, O. Schramm, and S. Sheffield, Markov chains in smooth Banach spaces and Gromov-hyperbolic metric spaces, Duke Math. J. 134 (2006), 165-197.

[106] A. Naor and G. Schechtman, Remarks on non linear type and Pisier's inequality, J. Reine Angew. Math. 552 (2002), 213-236.

[107] - Planar earthmover is not in $L_{1}$, SIAM J. Comput. 37 (2007), 804-826 (electronic).

[108] E. Odell and T. Schlumprecht, The distortion problem, Acta Math. 173 (1994), 259-281.

[109] J. Pelant, Embeddings into c $c_{0}$, Topology Appl. 57 (1994), 259-269.

[110] A. Pełczyński, Projections in certain Banach spaces, Studia Math. 19 (1960), 209-228.

[111] G. Pisier, Martingales with values in uniformly convex spaces, Israel J. Math. 20 (1975), 326350.

[112] _ Probabilistic methods in the geometry of Banach spaces, Probability and analysis (Varenna, 1985), 1986, pp. 167-241.

[113] G. Pisier and Q. Xu, Random series in the real interpolation spaces between the spaces $v_{p}$, Geometrical aspects of functional analysis (1985/86), Lecture Notes in Math., vol. 1267, Springer, Berlin, 1987, pp. 185-209.

[114] N. L. Randrianarivony, Characterization of quasi-Banach spaces which coarsely embed into a Hilbert space, Proc. Amer. Math. Soc. 134 (2006), 1315-1317 (electronic).

[115] Y. Raynaud, Espaces de Banach superstables, distances stables et homéomorphismes uniformes, Israel J. Math. 44 (1983), 33-52 (French, with English summary).

[116] M. Ribe, On uniformly homeomorphic normed spaces, Ark. Mat. 14 (1976), 237-244.

[117]__ On uniformly homeomorphic normed spaces. II, Ark. Mat. 16 (1978), 1-9.

[118] __ Existence of separable uniformly homeomorphic nonisomorphic Banach spaces, Israel J. Math. 48 (1984), 139-147.

[119] H. P. Rosenthal, On the subspaces of $L^{p}(p>2)$ spanned by sequences of independent random variables, Israel J. Math. 8 (1970), 273-303.

[120] I. J. Schoenberg, Metric spaces and positive definite functions, Trans. Amer. Math. Soc. 44 (1938), 522-536.

[121] S. J. Szarek, On the existence and uniqueness of complex structure and spaces with "few" operators, Trans. Amer. Math. Soc. 293 (1986), 339-353.

[122] M. Talagrand, Isoperimetry, logarithmic Sobolev inequalities on the discrete cube, and Margulis' graph connectivity theorem, Geom. Funct. Anal. 3 (1993), no. 3, 295-314.

[123] H. Toruńczyk, Characterizing Hilbert space topology, Fund. Math. 111 (1981), 247-262.

[124] I. G. Tsar'kov, Extension of Hilbert-valued Lipschitz mappings, Vestnik Moskov. Univ. Ser. I Mat. Mekh. (1999), 9-16, 72 (Russian, with Russian summary); English transl., Moscow Univ. Math. Bull. 54 (1999), no. 6, 7-14 (2000). 
[125] N. Weaver, Lipschitz algebras, World Scientific Publishing Co. Inc., River Edge, NJ, 1999.

[126] A. Weston, Some non-uniformly homeomorphic spaces, Israel J. Math. 83 (1993), 375-380.

[127] H. Whitney, Differentiable functions defined in closed sets. I, Trans. Amer. Math. Soc. 36 (1934), 369-387.

[128] G. Yu, The coarse Baum-Connes conjecture for spaces which admit a uniform embedding into Hilbert space, Invent. Math. 139 (2000), 201-240. 